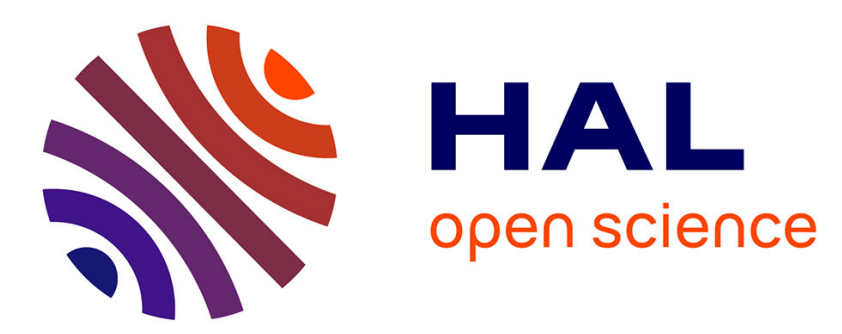

\title{
Lagrangian Methods in Experimental Fluid Mechanics
}

Mickaël Bourgoin, Jean-François Pinton, Romain Volk

\section{To cite this version:}

Mickaël Bourgoin, Jean-François Pinton, Romain Volk. Lagrangian Methods in Experimental Fluid Mechanics. Modeling Atmospheric and Oceanic Flows, insights from laboratory experiments and numerical simulations, 2014. hal-02634169

\section{HAL Id: hal-02634169 \\ https://hal.science/hal-02634169}

Submitted on 27 May 2020

HAL is a multi-disciplinary open access archive for the deposit and dissemination of scientific research documents, whether they are published or not. The documents may come from teaching and research institutions in France or abroad, or from public or private research centers.
L'archive ouverte pluridisciplinaire HAL, est destinée au dépôt et à la diffusion de documents scientifiques de niveau recherche, publiés ou non, émanant des établissements d'enseignement et de recherche français ou étrangers, des laboratoires publics ou privés. 


\title{
Lagrangian Methods in Experimental Fluid Mechanics
}

\author{
M. Bourgoin ${ }^{1}$, J.-F. Pinton ${ }^{2}$, R. Volk ${ }^{2}$
}

\begin{abstract}
Experimental techniques yielding measurement with high resolution in time and space domains have had a large impact in fluid mechanics over the past ten years. This review concentrates on Lagrangian approaches since the understanding of the motions of fluid particles is critical for transport phenomena, which play a major role in geophysical fluid dynamics. Applications range from mixing problems, passive and active scalar advection, to dispersion of particles, accretion or fragmentation. Experimental methods have been developed using several strategies: direct optical imaging to record the trajectories of tracer particles; scattering techniques (optics or acoustics) to track particle velocities and also probe density and vorticity fluctuations in the flow; remote sensing techniques to record dynamics of tracers and objects passively advected by fluid motions. These methods, underlying principles and main results are discussed here.
\end{abstract}

\section{Introduction}

Atmospheric and oceanographic flows are characterized by a strong complexity: stratification, anisotropy, global rotation, inhomogeneity, turbulence, etc. are just a few of the main features involved. This chapter reviews some of the most recent advances in metrology relevant to investigate such complex flows in model laboratory experiments. We focus on high resolution techniques which give access to the hierarchy of multiple spatial and temporal scales involved in this type of highly turbulent flows. As described below, among these techniques, Lagrangian approaches (where tracer particles are tracked in the flow) have been undergone significant developments in the past decade. Lagrangian metrology has become one of the most versatile and accurate tool for the investigation of complex flows, particularly suited in regards to geophysical motivations, where transport issues are paramount.

In the last decades, the increase of performance in numerical simulations of fluid dynamics processes (CFD) has motivated an increasing demand in the accuracy of models and, naturally, experimental measurements. A particular challenge in the context of atmospheric and oceanographic research concerns the improvement of the multi-scale description of flows and of energy cascade mechanisms. Geophysical flows are indeed characterized by a high turbulence intensity which results in an important hierarchy of relevant scales, with structures ranging from kilometers down to millimeters. In turbulent flows, the range of relevant scales (called the inertial range) between the energy injection scale $L$ and the dissipative scale (also called Kolmogorov scale) $\eta$ is directly related to the Reynolds number of the flow, Re: $L / \eta \propto R e^{3 / 4}$ [Tennekes and Lumley, 1992]. Reynolds numbers of the order $10^{6}$ are usual in geophysical flows, implying that at least 4 decades of spatial dynamics are typically involved. Similarly in the time domain, the ratio between the eddy turnover time $T_{L}$ at injection scale and at dissipation

${ }^{1}$ Laboratoire des Écoulements Géophysiques et Industriels, CNRS/UJF/GINP - Université de Grenoble, BP53 - 38041 Grenoble Cedex 9, France..

${ }^{2}$ CNRS UMR5672, Laboratoire de Physique de l'École normale supérieure de Lyon, 46 Allée d'Italie, 69007 Lyon, France.

Copyright 2014 by the American Geophysical Union. 0148-0227/14/\$9.00 scales $\tau_{\eta}$ goes as $T_{L} / \tau_{\eta} \propto R e^{1 / 2}$, covering three decades of temporal dynamics. These dynamical ranges can be even further extended toward the largest scales due to inverse cascade mechanisms, which may become important in the atmosphere or the ocean, at scales where dynamics exhibits 2D-properties, where flow structures can extend over hundreds of kilometers. When it comes to investigate related physics in laboratory experiments, with a typical dimension of the order of $1 \mathrm{~m}$ and typical correlation time scale of the order of $1 \mathrm{~s}$, the investigation of a comparable hierarchy of scales pushes the smallest involved structures down to tens to hundreds of microns (or even smaller) in space with a typical time-scale of fractions of milliseconds. This stimulates a permanent effort in the experimental community, to develop measurements with increasing degrees of accuracy and resolution. Technological advances in high speed digital imaging, with the ability to record pictures with millions of pixels at rates exceeding thousands of frames per second have opened up a new era in experimental fluid dynamics and laboratory models of geophysical flows. In parallel, several innovative developments of scattering techniques (sound or light) have emerged. More recently, progress in embedded sensor and radio transmission technologies have lead to the development of instrumented particles which probe the flow as they are entrained by the local motions.

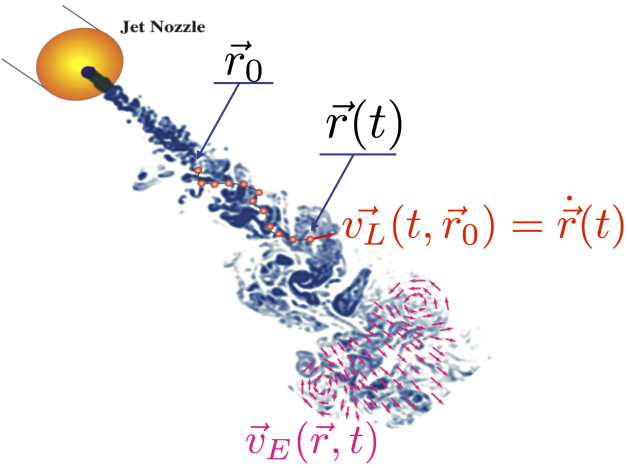

Figure 1. Eulerian versus Lagrangian description of a flow. In the Eulerian framework the flow is described in terms of the velocity field, while in the Lagrangian framework it is characterized from the trajectories of fluid tracers.

Let us briefly recall that flow measurements are done using two approaches : the Eulerian description and the La- 
grangian one (see figure 1). In Eulerian techniques, the fluid velocity $\vec{v}_{E}(\vec{r}, t)$ is studied as a field varying in space $\vec{r}$ an time $t$. Although this field also experiences in general instantaneous temporal fluctuations, in statistically stationary conditions, the time $t$ is generally considered as a parameter which helps building ensemble averages by repeating the measurement (if the system is not statistically stationary, then time $t$ becomes an actual variable of the problem which accounts for non-stationary effects). Eulerian measurements have for long been the most widely used in experimental fluid mechanics: hot-wire measurements, particle image velocimetry (PIV), laser Doppler velocimetry (LDV) are classical techniques. In the Lagrangian approach, instead of probing the flow at given fixed points $\vec{r}$ (where fluid particles constantly pass), velocity is measured along the path of given fluid elements which are tagged and tracked individually. In this representation, the velocity $\vec{v}_{L}\left(t, \vec{r}_{0}\right)$ changes in the course of time $t$ while space coordinates simply parametrize the initial position $\vec{r}_{0}$ of the fluid particle under investigation. In statistically homogeneous conditions, $\vec{r}_{0}$ is mainly a parameter which is considered either to improve statistical convergence by simultaneously tracking several particles with different initial separations or to address multi-particle problems, as mixing and dispersion.

Eulerian measurements have prevailed in experimental fluid mechanics for decades. Recent progress in Eulerian measurements mainly concern the improvement of PIV systems, which are now commonly available in 3D-3C configuration (where the three components of the velocity field are measured in a full 3D volume of the flow - for instance using tomographic reconstruction), with an increased repetition rate (thanks to the newest high speed camera technologies) giving access to time resolved measurements.

On the other hand, Lagrangian measurements have now been developed to the point where Lagrangian particle tracking is among the most accurate fluid dynamics measurements. Several factors have contributed to what we can call the Lagrangian revolution: (i) The importance of Lagrangian approaches for modeling. The relevance of a Lagrangian description of the dynamics of a fluid in the context of mixing and transport phenomena has been realized since Taylor [Taylor, 1922] and Richardson [Richardson, 1926] (in particular for atmospheric and oceanic flows). It is now essential for the study of dispersion (pollutants, for instance). Modern stochastic models are best developed in the Lagrangian domain. (ii) Technological progress have given access to the ultra-fast recording and processing performance required for Lagrangian tracking. This led for instance to the first measurements of acceleration of fluid particles in turbulent flows over a decade ago [Voth et al., 2002;

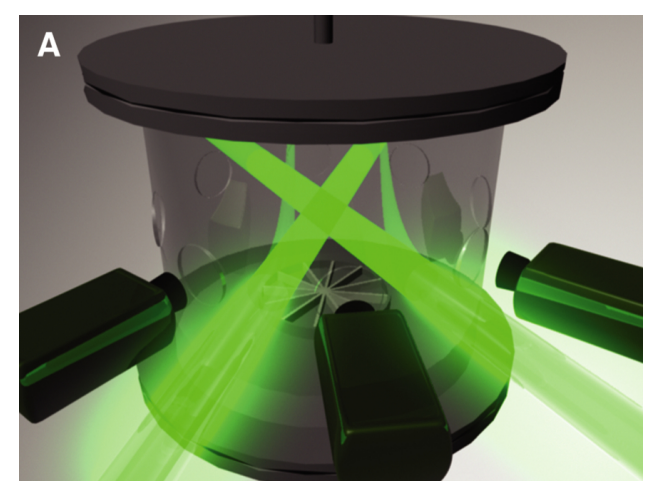

Figure 2. Sketch of a typical 3D-particle tracking experiment. The central part of the bulk of the flow is illuminated using two expanded high power laser beams. Three cameras record simultaneously the motion of small particle tracers in the bulk. Figure from [Bourgoin et al., 2006]
Mordant et al., 2001]. We detail in the following sections some of the latest measurement techniques used in state of the art laboratory experiments. We shall begin with optical techniques (Lagrangian tracking and Extended Laser Doppler Velocimetry (ELDV)), before moving to acoustical ones (Lagrangian tracking and vorticity measurement). We close this chapter with an introduction to instrumented particles. As it will be illustrated below, some of these techniques have already proven their relevance to investigate model flows with geophysical motivations (rotating fluids, stratified flows, turbulent transport phenomena, etc.).

\section{Optical techniques}

\subsection{Particle tracking}

An important advance in Lagrangian measurements has been done in 1997 by Virant and Dracos [Virant and Dracos, 1997] who developed a 3D-Particle Tracking Velocimetry (PTV) technique based on the direct imaging of small particles seeding the flow. They used simultaneously 4 video cameras at a rate of $25 \mathrm{fps}$ to access the $3 \mathrm{D}$ trajectories of several hundreds of particles at once. Ott and Mann [Ott and Mann, 2000] developed a similar technique to study relative dispersion of fluid particles. In those two pioneering experiments, because of the low frame rate, particle dynamics could be resolved only for flows at moderate Reynolds numbers (Re $<4000$ typically). LaPorta et al. [LaPorta et al., 2001], used silicon strip detectors (initially developed for high energy particles detection) at a frame rate up to $70 \mathrm{kHz}$, allowing the first fully resolved Lagrangian optical tracking measurements at Reynolds numbers approaching $10^{5}$. However, only one particle at a time could be tracked with the silicon strip detectors. Subsequently, Bourgoin et al. [Bourgoin et al., 2006] developed a high resolution 3DPTV facility similar to that of Virant \& Dracos and Ott \& Mann, but using ultrafast cameras at a repetition rate of $27 \mathrm{kHz}$, allowing the tracking of several hundred of particles in high Reynolds number regimes. In the context of atmospheric and oceanographic studies, similar 3D-PTV systems have been recently implemented to investigate for instance thermal convective flows [ $\mathrm{Ni}$ et al., 2012] as well as the influence of Coriolis force on the transport properties of rotating flows [Del Castello and Clercx, 2011]. We detail in the following how such multi-camera tracking systems work.

\subsubsection{Principle}

The principle of optical particle tracking is conceptually very simple: it consists in filming the motion of particles and thereafter to reconstruct their trajectories. However, its practical implementation is a challenge, and several aspects must be carefully considered:

Resolution issues. For high resolution measurements, high speed cameras with a large number of pixels are required. As already discussed, three decades of temporal resolution requires a repetition rate of at least $1 \mathrm{kHz}$ (assuming large structures evolve with a typical time scale around 1 second), while four decades of spatial resolution would in principle require a sensor with at least $10^{4} \times 10^{4}$ pixels. State of the art high speed cameras are typically capable to record $10^{3} \times 10^{3}$ pixel images at several thousands of frames per second, which yields over three decades in time and three decades in space. In practice, as discussed later, experimental noise generally requires to severely oversample the data, and this lowers the effective time resolution. On the other hand, several cameras are generally used simultaneously (as discussed below), which has the additional benefit to improve the effective resolution to about $1 / 10$ th of a pixel, hence recovering 4 decades of effective spatial 
resolution. The global resolution of a tracking system is generally a trade-off between temporal and spatial resolution, as higher repetition rates can be achieved by reducing the number of pixels and vice versa. However, thanks to the impressive progress in high speed digital imaging technology, direct optical tracking has become one of the most accurate techniques in experimental fluid mechanics.

3D issues. Complex flows generally involve 3D-structures which require tracking to be done in $3 \mathrm{D}$. This has two consequences: (i) the flow has to be illuminated in volume (a laser sheet, as done for instance in PIV, is not sufficient) and (ii) particles must be tracked in $3 \mathrm{D}$, hence requiring a stereoscopic configuration. In terms of illumination, as the tracers to be tracked are generally small (hence the diffused light is dim), and the repetition rate is high (hence exposure time is short) and the light beam is enlarged (to illuminate a volume), high power light sources are required. High power lasers have been generally used [Voth et al., 2002; LaPorta et al., 2001; Bourgoin et al., 2006], though alternative and less expensive solutions using high power LEDs start to be developed [Del Castello and Clercx, 2011]. In terms of recording, the stereoscopic reconstruction requires at least two cameras with two different angles of view to be used simultaneously. In practice three or more cameras are used. Increasing the number of cameras has two main advantages: (i) it allows to track more particles simultaneously, which is interesting to improve statistical convergence of the measurements, specially when multi-particle problems (for instance related to dispersion issues) are investigated ; (ii) the redundancy for particles which are seen simultaneously by more than two cameras, improves the accuracy of the 3Dpositioning of those particles, thus leading to an enhanced effective resolution. State of the art optical Lagrangian systems using three or four high speed cameras are capable of tracking several hundreds of particles with $1 / 10$ th of pixel of effective resolution. Figure 2 shows the three high speed cameras system implemented by Bourgoin et al. [Bourgoin et al., 2006].

Data management issues. High speed imaging experiments result in a huge data rate. For instance $1 \mathrm{kHz}$ acquisitions with three one megapixel sensors recording at a bit-depth of 8 bits, represent an effective data rate of a few Gygabytes per second of recording. These usually requires to couple the acquisition system to dedicated data storage and data processing servers.

The different steps of the data processing, essential for the optimization of the accuracy of the $3 \mathrm{D}$ tracking, are described next.

\subsubsection{Reconstruction of $3 \mathrm{D}$ trajectories}

Once the images of the tracers are recorded, the goal is to reconstruct the 3D trajectories of as many particles as possible. This operation requires three steps:

1. Particle detection: each image (at each time $t$ ) of each camera is analyzed to determine the position of the center of each visible particle. This step results in maps of the $2 \mathrm{D}$ position of the center of the particles on each frame of each camera.

2. 3D matching: the second step consists in combining at each given time $t$, the previous $2 \mathrm{D}$ maps of particle centers from the $N$ cameras in order to reconstruct (by stereomatching) the $3 \mathrm{D}$ position of the center of the particles, with the highest possible accuracy.

3. Lagrangian tracking: finally, once the 3D positions of particles are found for all time steps, an appropriate tracking algorithm allows to reconnect the trajectories.

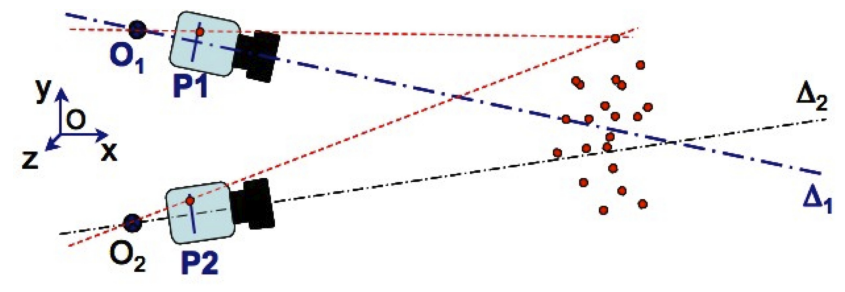

Figure 3. Optical tracking: 3D matching procedure see text.

We describe briefly the key points of the previous steps in the following paragraphs. Further details and useful information can be found in [Ouellette et al., 2005].

Particle detection. Ouellette et al. [Ouellette et al., 2005] have tested different algorithms for the detection of particle centers in $2 \mathrm{D}$ images. The choice of the best algorithm is a compromise between computation time and quality of the detection. The latter is quantified by both the accuracy with which the position of the center of the particles is determined and the number of particles correctly detected. The first step is to identify the local maxima of intensity on the image, indicating the presence of a particle. Then, the image around each maximum is analyzed to determine to the best accuracy the location of the center of the particle. For small particles (as generally used to seed the flow with tracers), the image of each individual particle does not exceed a few pixels. Under these conditions, simple algorithms based on the center of mass of intensity around the maximum are not sufficiently accurate. Algorithms based on neural networks can be very accurate, especially when images are very noisy, but relatively slow. A good compromise consists in fitting the local intensity profile by two Gaussians (one vertical and one horizontal), whose maxima define the center of the particle. The choice of two 1D Gaussian fits is preferred to that of one single 2D Gaussian because it is computationally significantly more efficient for almost the same accuracy. Ouellette et al. have shown that this method was typically capable of detecting $95 \%$ of particles and determing their position with sub-pixel accuracy.

3D matching. While the detection of particles can be made in the image space of each camera, 3D positioning and Lagrangian tracking must be made in real space (which is common to all cameras). The most widely used method to define the transformation for each camera between image space (in pixels) to real space (in real units), is based on a calibration method developed by Tsai [Tsai, 1987]. Each camera (let us say we consider camera $\# i$ ) is represented by a projection model defined by an optical axis $\Delta_{i}$, an optical center $O_{i}$ and a projection plane $P_{i}$. The image of a particle $X$ on the sensor of camera $\# i$ is then simply given by the intersection of the line $O_{i} X$ with the plane $P_{i}$ (see figure 3 ). The model is generally defined by at least 9 parameters for each camera: 6 external parameters for the absolute position of each camera $\left(3\right.$ coordinates for $O_{i}$ and 3 angles for the orientation of the optical axis $\Delta_{i}$ ) and 3 internal parameters (the distance $O_{i} P_{i}$, a coefficient for geometrical aberrations and the aspect ratio of the pixels). Refinement of this basic model can be considered, for instance by including several aberration coefficients (transverse and longitudinal). The parameters of the model are determined from the images of a calibration mask with known geometrical properties. Once the parameters of the model for each camera are determined, the $3 \mathrm{D}$ matching is performed as follows (see the illustration in figure 3 ): take the center of a particle $x_{i}$ as 
previously determined in pixels on the projection plane one of the cameras ; the real position $X_{i}$ of the particle in real space then lies somewhere of the line of view $O_{i} x_{i}$. The intersection of such line of views from two (or more) cameras defines the absolute 3D position of the particle in real space. In theory two cameras are sufficient to determine this intersection. In practice however, the lines of view rarely intersect due to slight imprecision in the calibration of the Tsai model. The 3D position is then defined as the point in real space which minimizes the distance to the different lines of view. Whenever a camera is added in the system, the redundancy of information provided by the additional line of view further restricts the possible $3 \mathrm{D}$ position of the particle. This greatly improves the effective spatial resolution of the 3D system. Ouellete et al. have shown that using three cameras instead of two gives an effective resolution of the order of one tenth "equivalent pixel" (that is to say one tenth of the spatial dimension whose image is the size of a pixel taking into account the magnification of the projective system). Thus the combination of three sensors of $10^{3} \times 10^{3}$ pixels provides an effective spatial resolution of 4 decades in $3 \mathrm{D}$. Adding a fourth camera, is then essentially interesting to increase the number of particles actually followed. Indeed, ambiguous situations where a particle hides another one in the line of sight of a camera may occur. These ambiguities can be lifted by adding a fourth camera at a different angle, in order to maximize the number of particles which are seen at any time by at least three cameras.

Trajectory reconnections. Lagrangian tracking consists in reconnecting particle trajectories between successive time steps. This requires to identify at time $t+1$ particles already detected at time $t$. Lagrangian tracking algorithms are generally based on the minimization of a given cost function. The simplest algorithm, called nearest neighbour simply consists in connecting a particle (let us say particle $\# j)$ whose position at time $t+1$ is $\vec{x}_{j}(t+1)$ to the particle $\# i$ whose position at time $t$ minimizes the cost function $\phi_{i j}=\left\|\vec{x}_{i}(t)-\vec{x}_{j}(t+1)\right\|$. This simple algorithm is accurate only if the inter frame displacement is significantly less than the average inter particle separation. It is therefore generally limited to relatively diluted configurations. In higher seeding density situations, more sophisticated algorithms are required. As shown by Ouellette et al., one robust algorithm consists in defining a cost function $\phi_{i j}$ based on four consecutive images. Qualitatively, it is based on a smoothest acceleration criterion. Quantitatively it is implemented as follows: assume trajectories has been reconnected up to time step $t$; the velocity of the particles is estimated from positions $\vec{x}_{i}(t)$ and $\vec{x}_{i}(t-1)$, which allows one to estimate their probable position at time $t+1, \overrightarrow{\tilde{x}}_{i}(t+1)$; then the particle acceleration is estimated from $\vec{x}_{i}(t-1), \vec{x}_{i}(t)$ and $\overrightarrow{\tilde{x}}_{i}(t+1)$ and this propagates at time $t+2$ an estimation $\overrightarrow{\vec{x}}_{i}(t+2)$ of the position for each particle in the vicinity of $\overrightarrow{\tilde{x}}_{i}(t+1)$; then the most probable trajectory is the one which minimizes the cost function $\phi_{i j}=\left\|\overrightarrow{\tilde{x}}_{i}(t+2)-\vec{x}_{j}(t+2)\right\|$.

\subsubsection{Example of 3D optical tracking}

Figure 4a shows an example of tracking of pairs of particles by Bourgoin et al. [Bourgoin et al., 2006] in the high Reynolds number experiment previously shown in figure 2 . The figure only shows two trajectories, but hundreds of such trajectories are simultaneously reconstructed. This allows a rapid statistical convergence of particle displacement, velocity and acceleration statistics. Such data can be used to investigate different properties of the flow. In the study by Bourgoin et al. separation statistics are investigated in order to address the longstanding question of turbulent super diffusion. But time resolved trajectories can be differentiated with time, once to obtain particle velocity and twice to access particle acceleration. Optical tracking of small particles has shown the highly intermittent dynamics of such
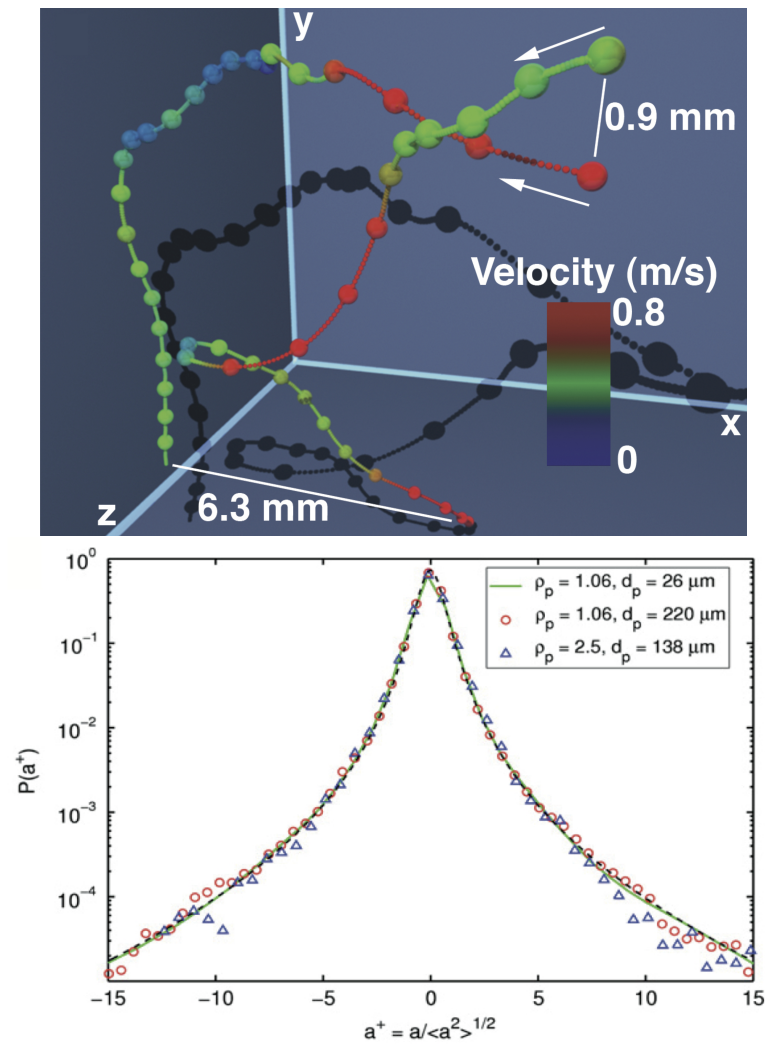

Figure 4. Top: Example of high resolution reconstruction of particle trajectories in experiment shown in figure 2. The small spheres mark every other measured position of the particles and are separated by $0.074 \mathrm{~ms}$ ; the large spheres mark every 30th position. Color indicate the velocity of the particle along its trajectory. (Figure from[Bourgoin et al., 2006]). One component acceleration statistics measured in a von Kármán swirling flow seeded with particles of different size and density (figure from [Xu and Bodenschatz, 2008])

fluid tracers in turbulent flows. This is revealed for instance by measurements of acceleration statistics in von Kármán swirling flows (shown in figure $4 \mathrm{~b}$ ) which exhibit highly nonGaussian fluctuations corresponding to events of very high acceleration occurring with a probability orders of magnitude larger than what would be expected for a normal random process with equivalent variance.

\subsection{Extended Laser Doppler Velocimetry}

As already mentioned, particle tracking is very demanding in terms of acquisition frequency which needs to be much larger than the inverse of the Kolmogorov timescale $1 / \tau_{\eta}=\sqrt{\epsilon / \nu}$ with a spatial resolution comparable with the Kolmogorov scale $\eta=\left(\nu^{3} / \epsilon\right)^{1 / 4}$ in order to access the very small scales of the particles motion. For typical water flows at the Lab scale one therefore needs to track particles with sizes in the range 10-100 microns with a sampling frequency larger than several kilohertz, which is a severe limitation in terms of camera specifications and cost of the experiment. To increase the temporal resolution at a modest cost, one possibility is to rely on scattering techniques using a reference wave (either using ultrasound or Laser light) that will be scattered by the moving particles. This is the basis of the so-called Laser Doppler Velocimetry (an Eulerian measurement technique), and of Extended Laser Doppler Velocimetry which is its extension to Lagrangian measure- 
ments.

Principle of Laser Doppler Velocimetry. The principle of Laser Doppler Velocimetry is quite simple: it uses two coherent laser beams (with wavelength $\lambda_{0}$ ) intersecting with an angle $\theta$ to create an interference pattern consisting in fringes perpendicular to the plane of the beams laser beams, separated by a distance $a=\lambda_{0} /(2 \sin (\theta / 2))$. When a particle crosses the fringes, it scatters light with an intensity $I(t)$ modulated with a frequency $f_{p}=u_{\perp} / a$, were $u_{\perp}$ is the component of velocity perpendicular to the fringes (figure ). If the measurement volume (region were the beams intersect) extends over a large region of space, a continuous detection of the instantaneous frequency $f_{p}(t)$ gives access to the evolution of the particle's velocity as a function of time. Such an Extended Laser Doppler Velocimetry was developed by Volk and coworkers [Volk et al., 2008, 2011] to perform velocity tracking of small particles in high Reynolds number flows.

Optical arrangement. In order to obtain interference fringes in a large region of space, only one laser beam is used: it is separated by a beam-splitter into two coherent beams separately expanded using two pairs of lenses as telescopes. The sign of the velocity can be extracted if
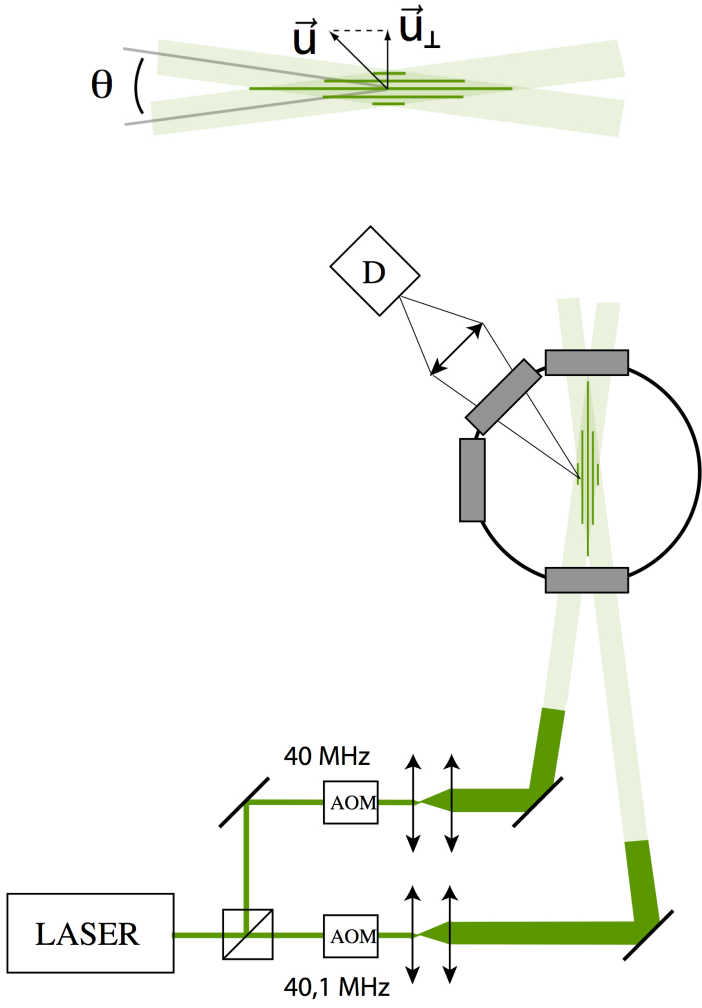

Figure 5. Optical arrangement of Extended Laser Doppler Velocimetry detailed in [Volk et al., 2008, 2011]. Two laser beams (with wavelength $\lambda_{0}$ ) forming an angle $\theta$ intersect to create an interference pattern with fringe spacing $a=\lambda_{0} /(2 \sin (\theta / 2))$. Each beam is shifted in frequency with an Acousto-Optic Modulator and expended using a telescope. The measurement volume is imaged onto a photodetector. A particle crossing the fringes scatters light modulated at frequency $f_{p}(t)=\delta f+u_{\perp}(t) / a$ with $\delta f$ the frequency shift between the beams and $u_{\perp}(t)$ the component of velocity perpendicular to the fringes. one creates a set of traveling interference fringes. This is achieved by shifting one of the optical beams at a frequency $\delta f$ so that the actual modulation of the scattered signal is at frequency $f_{p}(t)=\delta f+u_{\perp}(t) / a$. Shifting is down by propagating the the two laser beams through Acousto-Optic Modulators (AOM) with frequency shifts $f_{1}=40 \mathrm{MHz}$ and $f_{2}=40.1 \mathrm{MHz}$ so that the fringes are actually moving at constant velocity $v_{f}=a\left(f_{2}-f_{1}\right)=a \cdot \delta f$.

Particle detection. In practice, the intensity needed for the measurement depends on the particles used as tracers of the flow motion. Using a $1 \mathrm{~W}$ continuous Argon laser with wavelength $514 \mathrm{~nm}$ and a small angle between the beams, one can obtain a fringe spacing $a=41 \mu \mathrm{m}$. This is much larger than in classical LDV applications and allows to use as tracers small polystyrene fluorescent (with size $30 \mathrm{mi}-$ crons) or larger non fluorescent particles, which are almost neutrally buoyant in water. For the fluorescent particles case, scattered light is weaker and the measurement volume has to be imaged on a low noise photomultiplier with high gain. For particles larger than 100 microns, scattered signal is stronger and the detection can be made using amplified photodiodes and using less than $0.5 \mathrm{~W}$ of Laser power. As opposed to fluorescent particles, the optical contrast of the scattered signal strongly depends on the photodetector location and particle size, the detector is located in the plane of the beams and at 45 degrees from the beams.

Signal acquisition. The use of two AOM instead of one (for classical LDV) allows for a small frequency shift (100 $\mathrm{kHz}$ ) so that raw data can be acquired using high speed DACQ. Each time a particle crosses the measurement volume, it produces a burst of light with signal of the following form (figure 6(a)):

$$
\begin{gathered}
s(t)=\alpha(t)+\beta(t) \cos (2 \pi \delta f \cdot t+\phi(t)), \\
\text { with } \quad \frac{d \phi(t)}{d t}=2 \pi \frac{u_{\perp}(t)}{a},
\end{gathered}
$$

where $\alpha(t)$ and $\beta(t)$ are slowly varying envelopes originating from the Gaussian radial profiles of the beams.
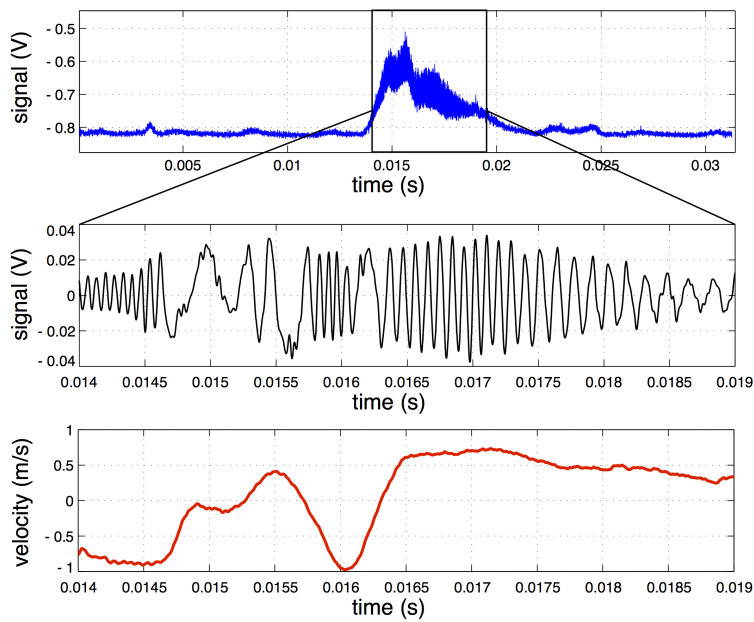

Figure 6. Typical optical signal measured with Extended Laser Doppler Velocimetry. Top : burst observed when a particle crosses the measurement volume. Middle : real part of the complex signal obtained signal after filtering and demodulation from carrying frequency $\delta f=100 \mathrm{kHz}$. Bottom : corresponding evolution of velocity as obtained from parametric estimation. 
$\nu(t)=\frac{1}{2 \pi} \frac{d \phi(t)}{d t}$ represents the Doppler shift of the scattered signal, due to the motion of the scatterer particle. In a typical situation, the diameter $d$ of the beams is much larger than the fringe spacing $a$ so that there is a scale separation between the fast modulation at frequency $\nu(t)=u_{\perp}(t) / a$ and the slow amplitude modulations $\alpha(t)$ and $\beta(t)$.

Signal processing. After running the experiment, the velocity is computed from the collection of light scattering signals $\left(s_{i}(t)\right)_{[1, N]}$. This signal processing step is crucial as both time and frequency - i.e. velocity - resolutions rely on its performance. As the local frequency of the signal is varying in time, common time-frequency techniques based on Fourier analysis [Flandrin, 1998] (such as short time Fourier transform are usually too limited as the Heisenberg principle imposes that time resolution $\delta_{t}$ and frequency resolution $\delta_{\nu}$ must comply the inequality $\delta_{t} \delta_{\nu}>1$, which means that one cannot have both high resolution in time (which is crucial to resolve the fastest dynamics of the particles) and frequency (which is crucial to have a good measurement of particles velocity, which according to relation (2) is directly given by $\nu(t))$. It is therefore necessary to overcome the Heisenberg principle limitation. Several methods exist, including Cohen class energetic estimators (such as Wigner-Ville and Choï Willians distributions) [Flandrin, 1998] which can be further refined using the reallocation technique [Flandrin, 1998; Kodera et al., 1976]. These methods are relatively time consuming in terms of computational processing, and are generally adapted for situations where no information is a priori known on the form of the signal to be analyzed. In order to increase the frequency resolution with small observation window, Mordant and coworkers introduced a fast demodulation algorithm with parametric estimation [Mordant et al., 2002, 2005]. It relies on a comparison between the measured signals $\left(s_{i}(t)\right)$ and the model given in equation (2). In practice, such a parametric estimate of amplitude and frequency modulations is very robust with respect to the experimental noise. The estimation is done in several steps :

1. As the timescale of $\alpha(t)$ (of order $d / u \sim 5 \mathrm{~ms}$ ) is very large as compared to $1 / \delta f=0.01 \mathrm{~ms}$, it is removed with high pass filtering at several kiloHertz.

2. To obtain an absolute definition of the local frequency through the evolution of the phase $\phi(t)$, the filtered signal $s^{\prime}(t)$ is transformed into an analytical complex signal $\underline{x}(t)$ : this is done using the Hilbert transform $\operatorname{HT}\left[s^{\prime}\right](t)$ of the measured signal with the definition $\underline{x}(t)=s^{\prime}(t)+i \mathrm{HT}\left[s^{\prime}\right](t)$. The amplitude and frequency of the signal are then $\alpha(t)=\|\underline{x}(t)\|$ and $f_{p}(t)=\delta f+d \phi / d t$ respectively.

3. The complex signal $\underline{x}(t)$ is then demodulated from the carrying frequency by multiplication by $\exp (-2 i \pi \delta f \cdot t)$. The real part of such demodulated complex signal for a typical burst is displayed in figure 6(middle).

4. For a fast and precise measurement of the modulation frequency, an approximated maximum likelihood (AML) method is coupled with a Kalman filter, so as to perform a parametric estimation of the instantaneous amplitude and frequency. In a moving window of duration $\delta T$, centered at time $t$, it assumes that the signal is made of a modulated complex exponential plus Gaussian noise $n(t)$, and compares the measured signal $\underline{x}(t)$ to the functional form

$$
\underline{z}(t)=A(t) e^{i 2 \pi \int_{0}^{t} \nu\left(t^{\prime}\right) \mathrm{d} t^{\prime}+i \psi}+n(t),
$$

were $A(t)$ and $\nu(t)$ are the unknown amplitude and frequency to be estimated and $\psi$ a constant phase originating from the initial particle position in the measurement volume. As an output, one obtains for each trajectory an estimate of the frequency $\nu(t)=u_{\perp}(t) / a$, amplitude $A(t)$, plus a confidence criterion $h(t)$ which measures the quality of the estimation at each time step. This is done for each trajectory on a Personal Computer using Matlab to obtain a collection of trajectories to be further used to compute Lagrangian statistics of the flow.

Initially designed for acoustical Doppler measurements (described in the next subsection), the demodulation technique proved to be fast and accurate enough to perform Lagrangian ELDV measurements with typical time resolution of $10 \mu \mathrm{s}$ and a sampling rate of $300 \mathrm{kHz}$. This represents the highest sampling rate ever used for Lagrangian measurements in high Reynolds number flows. It is particularly adapted to investigate flows with rapid and intense mutliscale swirling structures. Therefore, although this technique has never been used (to our knowledge) in experiments with geophysical motivations, it is very likely to be a good candidate for the investigation of most extreme atmospheric events.

Particle seeding issues. For practical applications, the particles seeding density has to be adjusted in order to be low enough so that one does not observe events with two particles at the same time in the measurement volume, but high enough to observe several trajectories per second. For a fully turbulent flow with Reynolds number at Taylor microscale $R e_{\lambda} \sim 600$, a collection of 15000 trajectories with mean duration 20 Kolmogorov times $\left(\tau_{\eta}\right)$ is enough to converge velocity statistics, acceleration statistics and acceleration autocorrelation function. In the case of acceleration
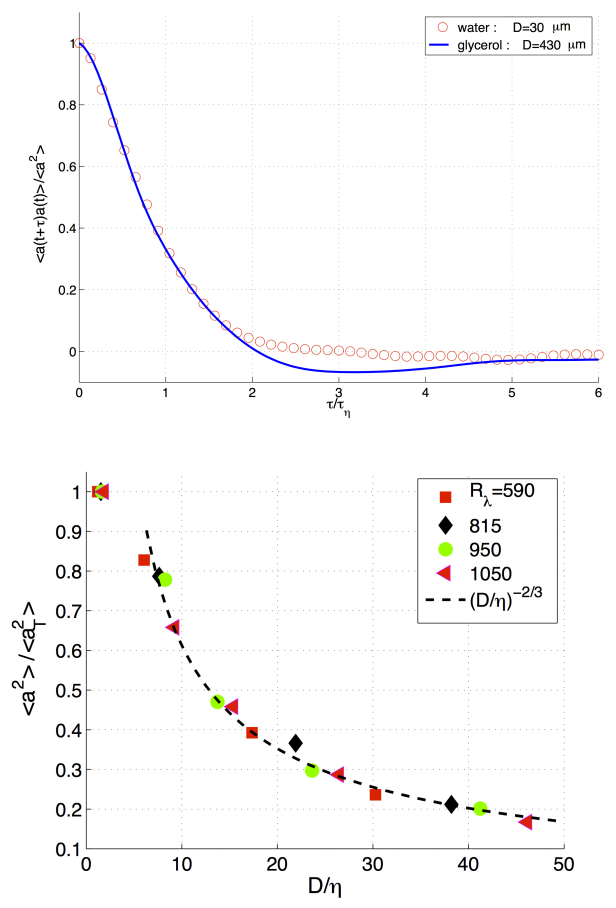

Figure 7. Top : Acceleration autocorrelation function $C_{a a}(\tau)=\langle a(t) a(t+\tau)\rangle /\left\langle a^{2}\right\rangle$ measured with ELDV in a fully developed turbulent flow. For both curves, time has been rescaled by the dissipative time $\tau_{\eta}=\sqrt{\nu / \epsilon}$. (०) $30 \mu \mathrm{m}$ fluorescent tracer particles for a water flow with dissipation scale $\eta=19 \mu \mathrm{m}$. (-) large $430 \mu \mathrm{m}$ polystyrene particles behaving as tracers in the same (water-glycerol) flow with dissipation scale $\eta=90 \mu \mathrm{m}$. For the two situations, the large scale driving and dissipation $\epsilon=20$ $\mathrm{W} / \mathrm{kg}$ are the same. Bottom : Acceleration variance of particles with size $D$ normalized by the one measured for tracers as a function of the ratio $D / \eta$. Particles with size $D / \eta>5$ no longer behave as tracers of the flow motions. 
autocorrelation $C_{a a}(\tau)=\langle a(t) a(t+\tau)\rangle /\left\langle a^{2}\right\rangle$, one gets access to an estimate of the local kinetic energy dissipation $\epsilon$ because the integral of the positive part of the curve is very close to the dissipation time $\tau_{\eta}=\sqrt{\nu / \epsilon}$ [Volk et al., 2011]. As shown in figure 7(top), there is a good rescaling between the acceleration autocorrelation functions when time is measured in $\tau_{\eta}$ units for fully developed turbulent flows of water (with kinematic viscosity $10^{-6} \mathrm{~m}^{2} / \mathrm{s}$ and dissipation scale 19 microns) and water-glycerol mixtures (kinematic viscosity $8 \cdot 10^{-6} \mathrm{~m}^{2} / \mathrm{s}$, dissipation scale 90 microns). These two curves also show that particles with diameters $D=5 \eta$ (the dissipative scale) can still be considered tracers of the flow. As shown in 7(bottom), this is no longer the case for larger particles for which one observes a decrease of particle acceleration variance following a power law $\left\langle a_{D}^{2}\right\rangle /\left\langle a_{\text {tracer }}^{2}\right\rangle \propto(D / \eta)^{-2 / 3}$. This decrease of acceleration variance goes together with an increase of the particle acceleration autocorrelation time [Qureshi et al., 2007; Brown et al., 2009; Volk et al., 2011].

\section{Acoustic techniques}

Whenever a sound wave encounters an obstacle or inhomogeneity along its propagation path, it is deflected from its original course, a phenomenon called acoustic scattering. The scatterer can be either a material obstacle or a physical inhomogeneity such as temperature or velocity gradient, which creates a contrast of acoustic impedance and influences the propagation of sound. The properties of the scattered acoustic wave depends upon the frequency of incident wave, the shape and size of the obstacles as well as their velocity. It is possible to take advantage of these scattering features to probe the dynamics of fluids. We detail here two recent techniques based on acoustic scattering : (i) Lagrangian acoustic tracking, which exploits the Doppler shift of the wave scattered by moving particles ; (ii) acoustical measurements of vorticity, which exploits the scattering properties of eddies in a fluid (with no need of seeding the flow). These techniques are intrinsically related to acoustic scattering properties and differ from more classical ones based on echo and time-of-flight measurements.

\subsection{Acoustic Doppler Lagrangian tracking}

\subsubsection{Principle}

Acoustic Lagrangian tracking is based on the measurement of the Doppler shift of the acoustic wave scattered by a moving particle. Figure 8 (top) shows the principle of one-component ultrasonic Doppler velocimetry. An acoustic transducer emits a continuous ultrasonic wave at a given frequency $\nu_{0}$ with a propagating direction $\vec{n}_{0}$. Whenever a particle crosses the acoustic beam of the emitter, it scatters the acoustic wave. An acoustic receiver then listens to the scattered wave in a specifc direction $\vec{n}_{s}\left(\theta_{s}=\left(\vec{n}_{0} ; \vec{n}_{s}\right)\right.$ is the scatter angle). The intersection between the emitting and the receiving beams defines the measurement volume, where particles can be actually detected. Because the particles moves, the scattered wave is Doppler shifted an its frequency $\nu_{s}$ differs from $\nu_{0}$ so that

$$
\frac{\nu_{s}-\nu_{0}}{\nu_{0}}=\frac{\vec{V} \cdot\left(\overrightarrow{n_{0}}-\overrightarrow{n_{s}}\right)}{c}=-2 \frac{v_{/ /}}{c} \sin \left(\theta_{s} / 2\right),
$$

where $c$ is the speed of sound in the experimental conditions. For a given incident frequency $\nu_{0}$ and a given scatter angle $\theta_{s}$, the instantaneous frequency shift $\delta \nu(t)=\nu_{s}-\nu_{0}$ gives a direct measurement of the projection, $v_{/ /}$, of the particle velocity along $\vec{n}_{0}-\vec{n}_{s}$ (note that this is an algebraic measurement: the sign of $v_{/ /}$is given by the sign of the frequency shift). Hence, the continuous recording of the frequency shift $\delta \nu(t)$ gives a Lagrangian measurement of the velocity component $v_{/ /}(t)$ along the particles trajectory. It
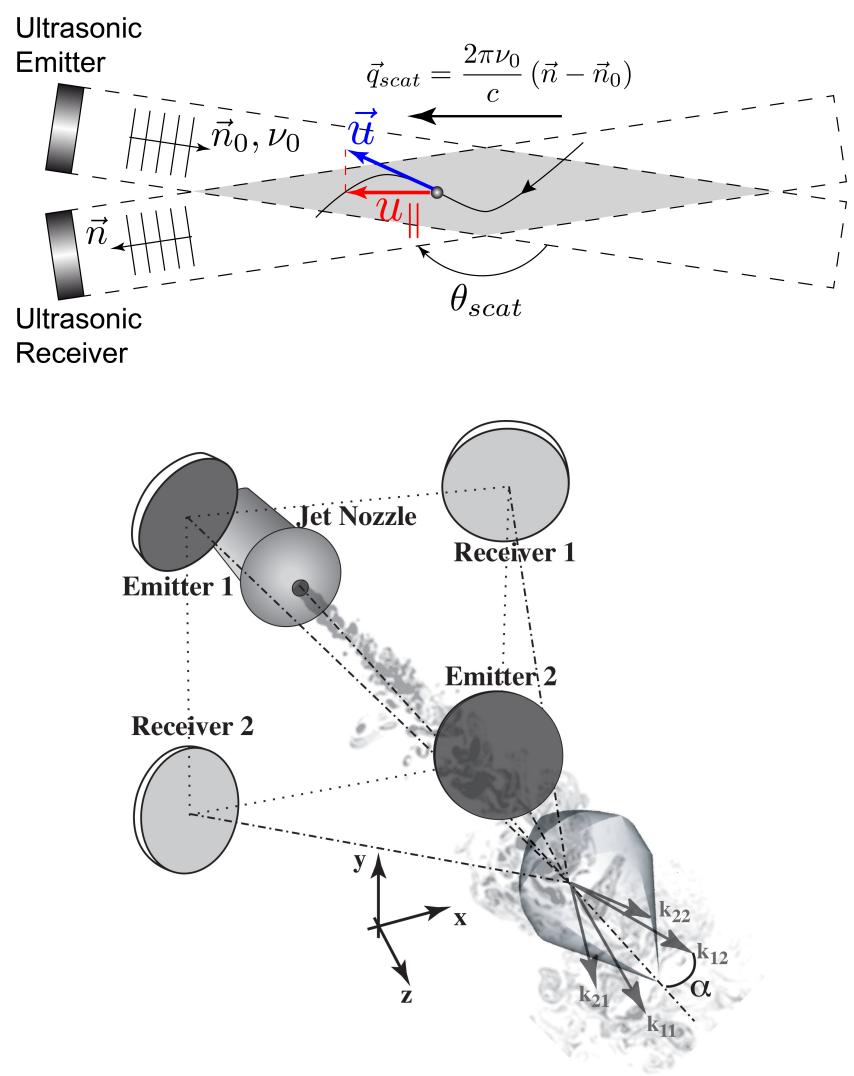

Figure 8. Top: Principle of one-component acoustic Doppler velocimetry. Note the analogy with the optical system used for the ELDV (see figure 5). Bottom: Example of transducer arrangement for the acoustic Lagrangian tracking of the three component of the velocity. Four transducers (two emitters and two receivers) are placed at the vertices of a square, tilted so that their axes cross at the same point on the jet axis, in a square-based pyramid configuration. The two emitter operate at two different working frequencies $\nu_{0,1}$ and $\nu_{0,2}$ and receivers listen to scattered waves in the vicinity of each of this frequencies. This arrangement is this composed of four independent pairs of emitter-receiver capable of measuring four projections of the velocity, which gives a redundant 3D measurement, where redundancy improves the signal to noise ratio (SNR). Adding extra transducers would increase further the SNR.

is interesting to note the analogy of this acoustic technique with the optical ELDV method previously described. The modulation of scattered light by a particle moving in the interference pattern in ELDV is indeed conceptually equivalent to the modulation of the Doppler shifted acoustic wave scattered by a particle in the present configuration. Finally, we point out that the combination of several pairs of transducers and working frequencies allows to extend the measurement and access two or three components of the velocity (see Figure 8 (bottom)).

Such an acoustic Lagrangian tracking technique was first implemented by Mordant et al [Mordant et al., 2001, 2002, 2005] in a pioneering study of Lagrangian turbulent statistics in a von Kármán swirling flow of water. In that case, piezo-electric elements were used as acoustic transducers (with typical emitting frequency operating in the $\mathrm{MHz}$ range) and small polystyrene particles served 


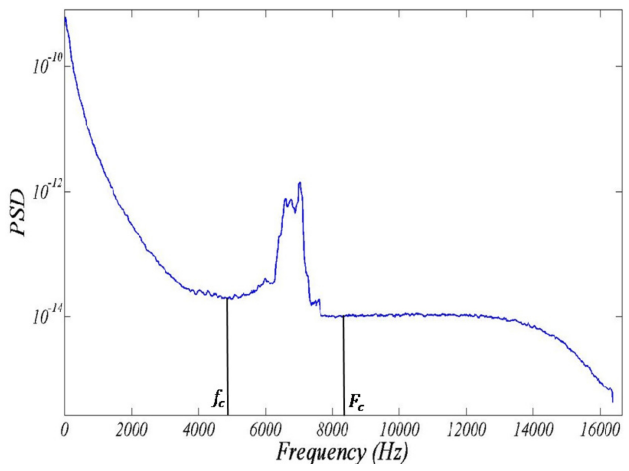

Figure 9. Typical spectrum of the donwmixed acoustic signal recorded by the receiver. The Doppler shift resulting from the scattering by moving tracers is visible around $7 \mathrm{kHz}$ (Figure from [Qureshi, 2009]).

as tracers. More recently, the same technique was ported to investigate opened air flows in a turbulent jet [Poulain et al., 2004] and wind-tunnel experiments [Qureshi et al., 2007, 2008]. It is interesting to note here that the same identical systems used by Poulain et al. and Qureshi et al. can also be easily ported to perform in situ measurements in real atmospheric flows. In these experiments Sell type acoustic transducers were used, operated with typical frequencies around $100 \mathrm{kHz}$ (ultrasounds at higher frequency are rapidly damped in air) and tracked particles were small milimmetric soap bubbles either neutrally buoyant (bubbles are then inflated with Helium) to have tracer behavior or intentionally heavier than air in order to address the question of the turbulent transport of inertial particles.

Pros and cons of scattering techniques compared to direct optical methods can be discussed. The main advantage of acoustics (shared with ELDV) is that Doppler shift measurements give a direct access to the tracer's velocity, while direct optical tracking requires to differentiate the position signal of the tracked particle to get velocity, an operation which is very sensitive to noise. Hence optical tracking usually requires severe oversampling if velocity and acceleration statistics are to be investigated. Other advantages of acoustic tracking are (i) the low cost of the required equipment, compared to expensive high speed cameras, (ii) the possibility to probe flows in opaque fluids (as liquid metals for instance), (iii) the possibility to easily explore large volumes and (iv) the ability to investigate open flows with large mean velocities. The last point is particularly important for instance in wind-tunnel or jet experiments where efficient optical tracking generally requires to mount the camera on a platform moving at the mean wind speed [Ayyalasomayajula et al., 2006] in order to track particles for sufficiently long times (typically comparable to the largest time scales of the flow) while, acoustic tracking can be efficiently done using fixed transducers ; moreover a backscattering configuration $\left(\theta_{\text {scatt }} \lesssim 180^{\circ}\right)$ allows to significantly extend the streamwise dimension of the measurement volume [Qureshi et al., 2007, 2008].

Among disadvantages, there is a strong limitation of acoustic tracking is its inability to accurately track several particles simultaneously. If more than one particle is present in the measurement volume, the signal recorded by the receiver superimposes the waves scattered by all the particles. Although signal processing strategies (discussed below) do exist to extract the contributions from each individual scatterer the accuracy decreases with increasing number of particles.

\subsubsection{Signal Processing and Doppler shift extraction}

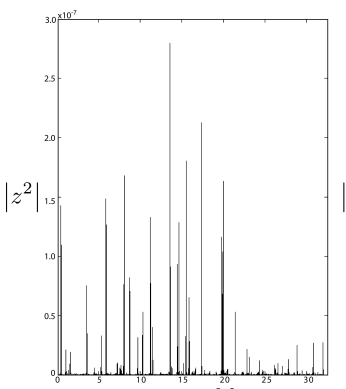

Time $[\mathrm{s}]$

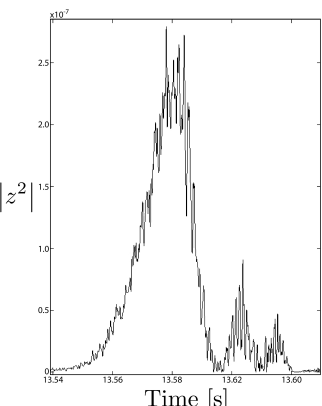

Time $[\mathrm{s}]$
Figure 10. (a) Typical amplitude of the recorded downmixed signal bandpass filtered around the Doppler peak (between frequencies $f_{c}$ and $F_{c}$ as shown in figure 9). Peaks of high amplitude correspond to events where a particle travels into the measurement volume and scatters the incident acoustic wave towards the receiver. (b) Zoom on one such isolated event. Figure from [Qureshi, 2009]).

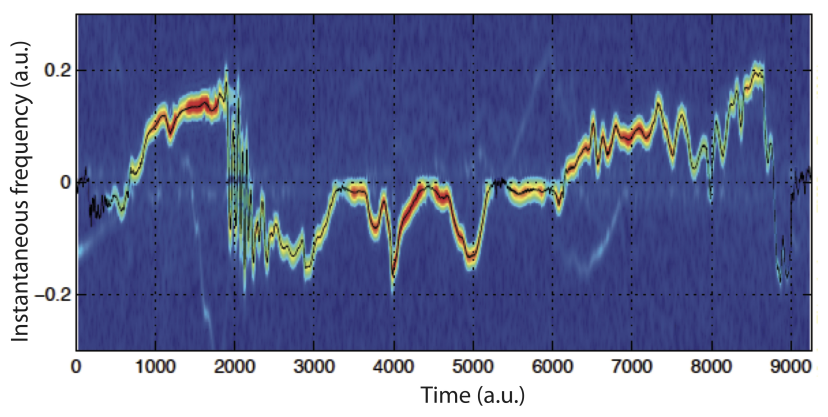

Figure 11. Example of reconstruction of the instantaneous frequency scattered by a moving sphere in a swirling flow of water. The color image represents the spectrogram computed with usual Fourier analysis timefrequency analysis (the width of the frequency trace illustrates the incertitude due to Heisenberg constrain) to which is superimposed the estimation given by the AML method (solid black line). Figure from [Mordant, 2001].

The acoustic signal recorded by the receiver combines a spectral component around the emitting frequency $\nu_{0}$, which corresponds to echoes and reflections directly incoming in the receiver without being scattered by the particles and a Doppler shifted component around $\nu_{d}$ resulting from the fraction of acoustic wave scattered by the moving particle. The information about particle velocities is entirely encoded in the Doppler shift $\delta \nu=\nu_{s}-\nu_{0}$. Therefore, a heterodyne downmixing is generally operated between the emitted and received signal which essentially results in shifting the emitting frequency $\nu_{0}$ to zero. Figure 9 shows a typical spectrum of a downmixed signal recorded in the wind-tunnel experiment by Qureshi et al. [Qureshi et al., 2007]. The peak at zero frequency corresponds to the emitting frequency $\nu_{0}$ (which was $80 \mathrm{kHz}$ in this experiment) and the secondary peak (around $7 \mathrm{kHz}$ ) corresponds to the Doppler shift from the wave scattered by moving tracers (note that the central peak at the emitting frequency is enlarged by aerodynamic effects). The interest of heterodyne down mixing is that recording the original signal would require very high sampling rates, as the Doppler frequency would be here around 

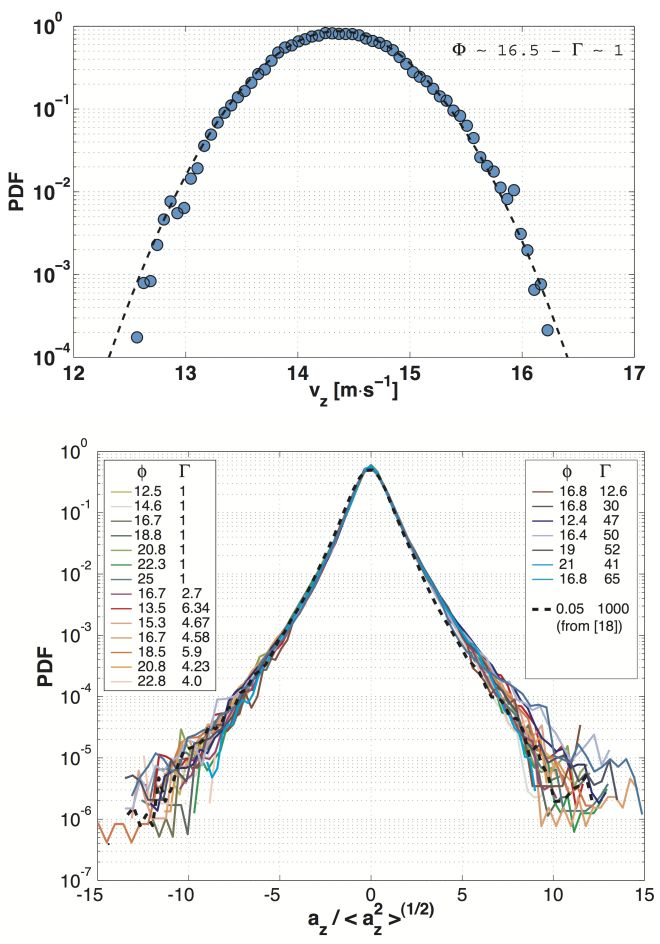

Figure 12. Top: One component Lagrangian velocity PDF of finized particles neutrally buoyant particles transported in a turbulent wind-tunnel flow. Figure from [Bourgoin et al., 2011]. Bottom: Acceleration PDF of finite size material particles with different sizes and density (parameters $\Phi$ and $\Gamma$ ) in the same wind-tunnel flow. Figure from [Qureshi et al., 2008].

$\nu_{s}=\nu_{0}+\delta \nu \simeq 87 \mathrm{kHZ}$ ), while it is only at $7 \mathrm{kHz}$ after downmixing.

Figure 10(a) shows the amplitude $A(t)$ of the down-mixed signal versus time; each peak of amplitude corresponds to the passage of a particle in the measurement zone. The spectrum previously discussed was calculated from the entire times series and hence all time information has been lost: the observed Doppler peak corresponds to the superposition of spectral contributions from thousands of successive scattered traveling through the measurement volume. It is only interesting to extract global informations, such as the average velocity of the particles (given by central frequency of the Doppler peak) or the typical level of velocity fluctuation (given by the width of the Doppler peak). However, accessing the Lagrangian dynamics of the particles requires to extract the instantaneous Doppler shift $\delta \nu(t)$ for each individual particle.

This is achieved in two steps: first, each event corresponding to the passage of a particle is detected from the amplitude signal as shown in figure 10(a) (figure 10(b) shows a zoom on such an individual particle event) ; second, the portion of signal corresponding to each individual event is analyzed with dedicated time-frequency tools to extract the instantaneous Doppler shift. For the same reasons previously discussed in the context of optical ELDV, the instantaneous Doppler shift can be efficiently extracted using an approximated maximum of likelihood (AML) algorithm where after down-mixing, the signal scattered by one particle and recorded by the receiver is modeled with the following form:

$$
z_{i}(t)=A_{i}(t) e^{i 2 \pi \int_{0}^{t} \delta \nu_{i}\left(t^{\prime}\right) \mathrm{d} t^{\prime}}+n(t)
$$

where $A_{i}(t)$ is the amplitude of the scattered acoustic wave, $\delta \nu_{i}(t)$ is the instantaneous frequency of the signal (subscript $i$ indicates that we consider particle number $i)$ and $n(t)$ represents an additive experimental noise. The AML algorithm determines for each particle $i$ the best functions $A_{i}(t)$ and $\delta \nu_{i}(t)$ so that the model given by eq. 9 matches as close as possible the actual recorded signal $(n(t)$ is assumed to be a random Gaussian noise whose amplitude is fixed according the actual noise level of the experimental signal). Such a parametric algorithm is capable of overcoming the Heisenberg limitation (which would severely constrain the resolution of the measurement), thanks to the extra information - given by the a priori imposed shape of the signal in eq. (9) added in the signal processing. The gain in resolution offered by the AML method is illustrated by figure 11 . Also of interest is the fact that the AML algorithm yields a quantitative indicator of the relevance of the expression (9) for the actual modeling of the scattering signal. This allows to discard from the statistical ensemble spurious events for instance when two particles were simultaneously present in the measurement volume.

\subsubsection{Example of acoustical particle tracking}

Acoustical tracking has been used in different experimental facilities, including von Kármán swirling flows, turbulent jets and wind-tunnels to investigate both the characteristics of the flow and the dynamics of material particles transported by the flow. Figure 12 represents the probability density functions of the velocity and acceleration of material particles transported in a turbulent wind-tunnel flow. Velocity is found to have Gaussian statistics while acceleration exhibit wide non-Gaussian tails, even for particles much denser than the fluid.

\subsection{Vorticity measurements}

Measuring vorticity has always been a challenge in experimental fluid mechanics, especially when small scales are to be probed. We recall here that the vorticity $\vec{\Omega}$ of a velocity field $\vec{u}$ is given by the curl of $\vec{u}(\vec{\Omega}=\vec{\nabla} \times \vec{u})$. A direct measurement of vorticity is usually done from spatial derivatives of the velocity field. This is typically the case with PIV or multiple hot-wire measurements. However, in either cases, the spatial resolution is an issue when flows are highly turbulent as neither PIV, nor multiple hot-wire probes are capable of resolving the smallest dissipative scales of the velocity fields, which are essential for an accurate estimation of a spatial derivative. We report in this section an elegant measurement of the vorticity of a flow based on the peculiar interaction between an acoustic wave and the vortical structures of a flow. In the context of atmospheric and oceanographic studies, this technique may be particularly suited to address questions related for instance to the interaction between vortices and the influence of global rotation as well as the turbulent cascade of enstrophy at mesoscales for which the atmosphere and/or the ocean can be considered as 2D.

\subsubsection{Principle}

The method relies on the interaction between an acoustic wave and the velocity gradients in the flow. The scattering properties from this acoustic-fluid interaction are nontrivial. Several theoretical and numerical studies can be found on the subject [Obukhov, 1953; Kraichnan, 1953; Chu, 1958; Batchelor, 1957; Lund and Rojas, 1989; Llewellyn Smith and Fort, 2001; Colonius et al., 1994]. In particular, using a Born approximation, Lund and Rojas [Lund and Rojas, 1989] have shown that the scattered amplitude of a plane acoustic wave by a turbulent flow can be linearly related to the spatial Fourier transform of the vorticity field of the flow. This property can be qualitatively understood as the fact that each vortex in the flow acts as a scatterer 


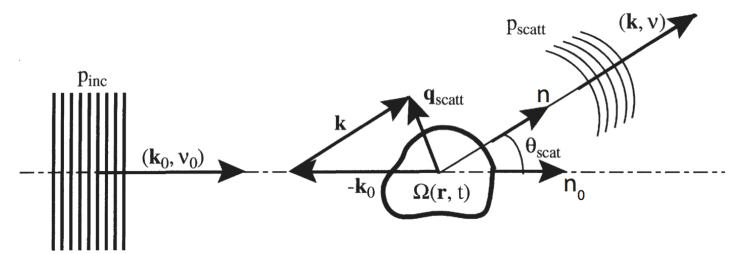

Figure 13. Typical implementation of acoustic scattering for probing the vorticity field of a flow. An ultrasonic emitter generates a plane acoustic wave in the direction $\vec{n}$ at the frequency $\nu_{0}$. A receiver listen to the acoustic wave scattered in a given direction $\vec{r}$. The relative amplitude of the scattered acoustic pressure to the incident acoustic pressure can be related to the spectral distribution of the vorticity field $\vec{\Omega}(\vec{x}, t)$ according to relation 6 . Figure from [Poulain et al., 2004].

which radiates a sound wave as it is perturbed by the incident impinging acoustic wave. The global scattered wave, results from the coherent average over the scatterers distribution. Figure 13 illustrates a typical acoustical scattering configuration which can be used to probe the vorticity of a flow. Lund et al. have shown that the acoustic pressure amplitude and the Fourier transform of the vorticity field are related as follows:

$$
p_{\text {scat }}(\vec{k}, t)=L\left(\theta_{\text {scat }}\right) \tilde{\Omega}_{\perp}\left(\vec{q}_{\text {scat }}=\vec{k}-\vec{k}_{0}, t\right) p_{\text {inc }}\left(\vec{k}_{0}, t\right),
$$

where $\vec{k}_{0}$ and $\vec{k}$ are the vector wave-numbers of the incoming and scattered acoustic waves respectively, $\theta_{\text {scat }}$ is the scattering angle, $p_{\text {inc }}$ and $p_{\text {scat }}$ are the complex pressure amplitudes of the incoming and scattered acoustic wave respectively, $L\left(\theta_{\text {scat }}\right)$ is an angular factor which will be discussed further below and $\tilde{\Omega}_{\perp}$ is the component of the space Fourier transform of the vorticity perpendicular to the scattering plane defined by the vector wave-numbers of the incident and scattered acoustic waves (see figure 13):

$$
\tilde{\Omega}_{\perp}(\vec{q}, t)=\left(\vec{n}_{0} \times \vec{n}\right) \cdot \iiint \vec{\Omega}(\vec{r}, t) e^{-i \vec{q} \cdot \vec{r}} \mathrm{~d}^{3} \vec{r} .
$$

Equation (6) therefore shows that the amplitude of the scattered wave gives a direct measurement of one Fourier mode of the vorticity component $\Omega_{\perp}$. Interestingly, the Fourier mode at which the vorticity field is being probed is directly selected by the imposed scattering vector $\vec{q}_{\text {scat }}=$ $\vec{k}-\vec{k}_{0}$. Hence, it is possible to reconstruct the complete vorticity spectra by spanning the explored scattering vector,and this can be done either by changing the angular position of the acoustic receiver or by changing the operating acous-

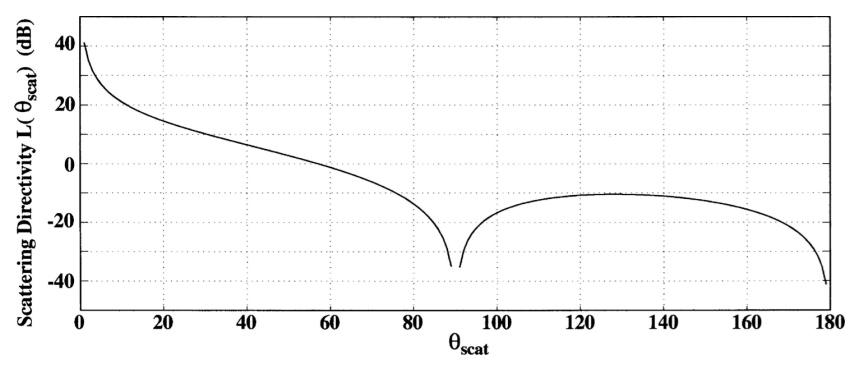

Figure 14. Angular factor $L\left(\theta_{\text {scat }}\right)$ Figure from [Poulain et al., 2004]. tic frequency (as $\left\|\vec{q}_{\text {scat }}\right\| \simeq 4 \pi \nu_{0} / c \sin \left(\theta_{\text {scat }}\right)$, assuming the Doppler shift $\nu-\nu_{0}$ remains small compared to $\left.\nu_{0}\right)$.

It is important to stress that this measurement is local in Fourier space, meaning that for a given scattering vector, only the mode of vorticity at wavenumber $\vec{q}_{\text {scat }}$ is actually measured. Hence the measurement is naturally global in space and the corresponding spectral mode is characterized across the entire measurement volume. Though the scattering structures of the flow are tracked as they move across the flow (this results for instance in a Doppler shift of the scattered acoustic wave) the recorded signal represents a coherent average of all structures at the probed scale simultaneously present in the measurement volume and no information is extracted from individual scatterers. As a consequence this technique is not properly speaking of Lagrangian type, although the instrumentation is almost identical to that described in the previous subsection on Lagrangian acoustical tracking.

An important point to be considered is the angular factor $L\left(\theta_{\text {scat }}\right)$ present in eq. (6). Figure 14 shows the dependency of $L\left(\theta_{\text {scat }}\right)$ with the scattering angle $\theta_{\text {scat }}$ as calculated by Lund et al.. It shows a quadrupolar like radiation pattern which diverges at zero angle (Born approximation fails in this limit) and vanishes for scattering angles $\theta_{\text {scat }}=90^{\circ}$ and $\theta_{\text {scat }}=180^{\circ}$ (back-scattering situation). Those two specific scattering angles are to be avoided for the vorticity measurement. On the contrary, they are optimal for particle tracking as no signal is then recorded from scattering effects of the fluid itself, and only the particles seeding the flow will be seen. This explains the back-scattering configuration chosen for the acoustical particle tracking described in the previous subsection.

\subsubsection{Experimental implementation and typical re-} sults

Experimental evidence of ultrasonic scattering by vortical structures in a flow has first been given by Baudet et al. [Baudet et al., 1991] in the canonical configuration of the von Kármán vortex street behind a cylinder at low Reynolds number. Since then, the technique has been ported to turbulent flows at moderate Reynolds number (in a turbulent jet of air [Poulain et al., 2004]) and at high Reynolds number (in a cryogenic turbulent jet of gaseous Helium [Bezaguet et al., 2002; Pietropinto et al., 1999]). Figure 15 shows

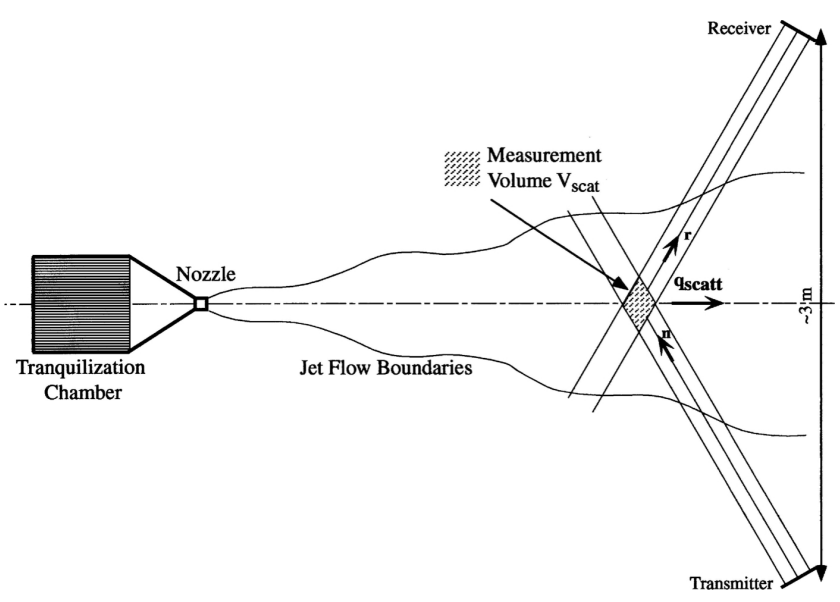

Figure 15. Exemple of implementation of vorticity measurement by acoustic scattering in a turbulent jet. Figure from [Poulain et al., 2004]. Note that the same configuration is can be easily ported to open flows and in city measurements in the atmospheric boundary layer for instance. 
the schematic of the implementation of acoustical measurement of vorticity in a turbulent jet as done by Poulain et al. [Poulain et al., 2004]. In this experiment acoustic transducers are of Sell type consisting of a circular plane piston, with typical diameter of the order of $10 \mathrm{~cm}$ (larger and smaller transducers can be used depending on the extent of the flow to be probed), made of a thin mylar sheet (typically $15 \mu \mathrm{m}$ thickness). One important advantage of such transducers is their large band-width (typically between $1 \mathrm{kHz}$ and $200 \mathrm{kHz}$ in air) which allows to span a wide range of scattering wave-vectors $\vec{q}_{\text {scat }}$ with a fixed geometrical arrangement (in particular with a fixed scattering angle). In the example, the scattering angle was kept fixed at constant value of the order of $60^{\circ}$. The choice of the working scattering angles responds to several criteria : (i) given the angular factor dependence shown in figure 14 angles close to $90^{\circ}$ and $180^{\circ}$ should be avoided; (ii) at small scattering angles the angular factor increases rapidly, however unless thermal conditions in the experiment are very well controlled small scattering angles should be avoided as forward sound scattering is very sensitive to temperature gradients; (iii) other practical criteria include for instance geometrical constraints around the experiment, limitation of echoing effects and direct acoustic "blinding" from the emitter to the receiver (in particular via the secondary diffraction side-lobes of the transducers); (iv) but beyond these practical considerations, the scattering angle should also be chosen in accordance to the physical properties of the flow to be probed. As already discussed the amplitude of the scattering vector $q_{\text {scat }}=4 \pi \nu_{0} / c \sin \left(\theta_{\text {scat }} / 2\right)$ defines the wave number at which the vorticity spectrum is being probed. It can be selected by changing either the working frequency or the scattering angle. Hence, the scattering angle will be chosen so that wave numbers relevant to the investigated problem can be effectively spanned within the accessible range of operating frequencies of the acoustic transducers. In our case, an angle of $60^{\circ}$ allowed the authors to probe a significant range of the inertial scales of the turbulent jet with a constant scattering angle by simply varying the working frequency $\nu_{0}$ of the transducers.

As an example of results which can be obtained with this technique, we show in figure $16 \mathrm{a}$ a typical power spectrum of the signal recorded by the acoustic receiver (note that the signal has been down-mixed exactly in the same way as explained for the acoustic Lagrangian measurement in the previous section). The maximum of the power spectrum occurs for a non-zero frequency which corresponds to the Doppler shift related to the average velocity of the jet at the location of the measurement volume. The range of detected frequencies around this maximum corresponds to the range of velocity statistically sampled by the vortices in the flow (within the range of spatial scales selected according to the wave-number $\left.q_{\text {scat }}\right)$ which scattered the acoustic wave. As a consequence, the shape of the power spectrum directly reflects the statistical properties of the velocity field of the flow. It is a Gaussian, centered around a frequency which corresponds to the mean stream velocity of the jet flow at the location of the measurement volume and the width of the Gaussian corresponds to the standard deviation of the carrier velocity field. Poulain et al. have indeed shown that in their turbulent jet the power spectrum is well fitted by a Gaussian :

$$
P S D_{\text {scat }}(\delta \nu)=\frac{A\left(\nu_{0}\right)}{\sqrt{2 \pi} \delta \nu_{r m s}} e^{-\frac{\left(\delta \nu-\delta \nu_{a v g}\right)^{2}}{2 \delta \nu_{r m s}^{2}}}
$$

and they have shown that the fitting Doppler shift frequencies $\delta \nu_{\text {avg }}$ and $\delta \nu_{r m s}$ where in excellent agreement with hotwire anemometry measurements of the mean and rms velocity of the jet flow. Perhaps more interestingly, they have shown that the quantity $q_{\mathrm{scat}}^{2} A\left(\nu_{0}\right)$ (where $A\left(\nu_{0}\right)$ is the maximum of the power spectral density) gives a direct estimate of the enstrophy spectrum of the flow at the given wavenumber $q_{\text {scat }}$, once the transfer function $H\left(\nu_{0}\right)$ between the receiver and the emitter is applied (an important aspect to be considered when using this methods concerns indeed the calibration of the acoustic transducers: while this is not crucial for the Lagrangian measurement previously described, which only relies on the frequency shift information of the scattered wave, a proper calibration of the transfer functions of the receiver and the emitter is required to extract the vorticity information, which is coded in the amplitude of the power spectral density). By varying the working frequency $\nu_{0}$ (and hence the scattering wave-number $q_{\text {scat }}$ ), it is then possible to reconstruct the entire spectrum of enstrophy. Figure $16 \mathrm{~b}$ shows the enstrophy spectrum of the jet flow investigated by Poulain et al. which is found to be in reasonable agreement with the Kolmogorov phenomenology of turbulence.
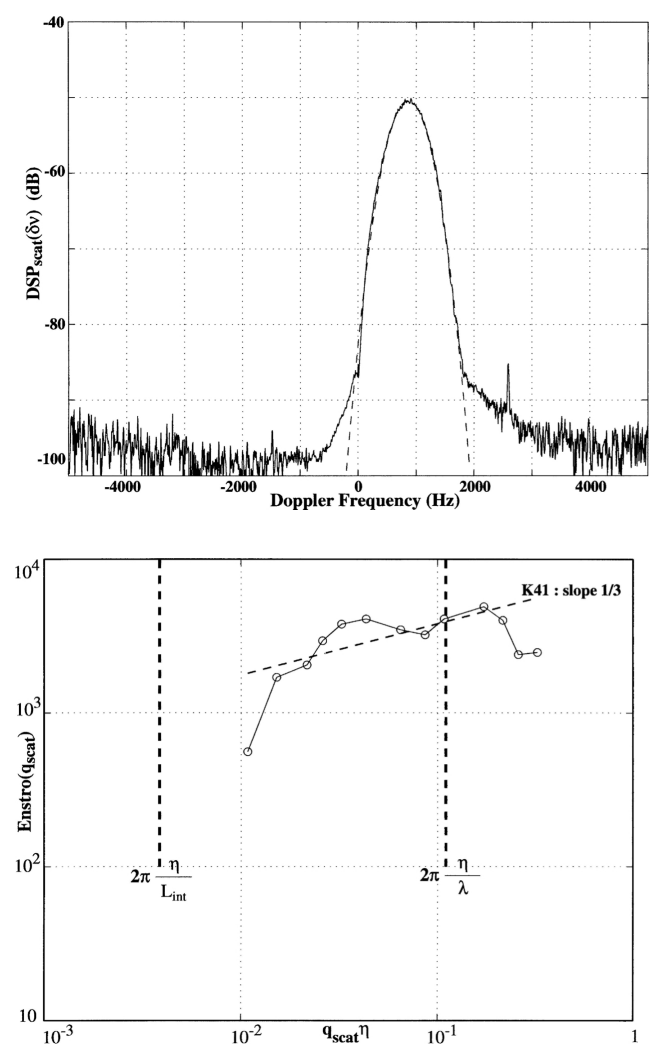

Figure 16. (a) Power spectral density of the signal scattered by the flow. (b) Discrete reconstruction of the spatial spectrum of the flow enstrophy. Figure from [Poulain et al., 2004].

\section{Instrumented particles}

When performing Lagrangian measurement in a fluid flow, one is usually limited in track length because of the necessary finite size of the measurement volume. Using Particle Tracking Velocimetry or Doppler Velocimetry one is also limited in the investigation of kinematic quantities (velocity, acceleration, vorticity, ...). Tracking scalar quantities such as salinity or temperature along particles path may also be of prime interest, in particular for stratified flows with geophysical motivations. One may therefore want to use instrumented particles (called 'smart particles') with embarked 
electronics able to measure scalar or kinematic quantities in Lagrangian frame while continuously transmitting the information to the operator for data storage and post-processing.

\subsection{Lagrangian temperature measurement}

A smart particle has been designed to measure continuously temperature along the particle trajectory using 4 thermistors, placed at the surface of the particle directly in contact with the fluid [Gasteuil et al., 2007; Shew et al., 2007]. It is made of a spherical capsule of diameter $D=21$ $\mathrm{mm}$ containing temperature instrumentation, a Radio Frequency (RF) emitter and a battery. It uses a resistance controlled oscillator LMC555 timer to create a square wave whose frequency depends on the temperature of the several thermistors. This square wave is used directly to modulate the frequency of the radio wave generated by the RF emitter in the range $[22-26] \mathrm{kHz}$ about the carrying frequency $f_{0}=315 \mathrm{MHz}$ (see figure 17 and reference [Shew et al., 2007] for more details). The entire mobile circuitry is powered with a coin cell battery which conditions the duration of the measurement, about 3 hours. Using an antenna and radio frequency $R F$ receiver and amplifiers, it is possible to acquire directly the demodulated signal oversampling with a high speed DACQ. The square wave signal frequency is then directly measured using standard Labview library, and time varying frequency converted to temperature using frequency-temperature calibration of the thermistors. The time resolution for this distant temperature measurement is about $10 \mathrm{~Hz}$ which was suitable for instance for studying turbulent thermal convection in laboratory experiments.

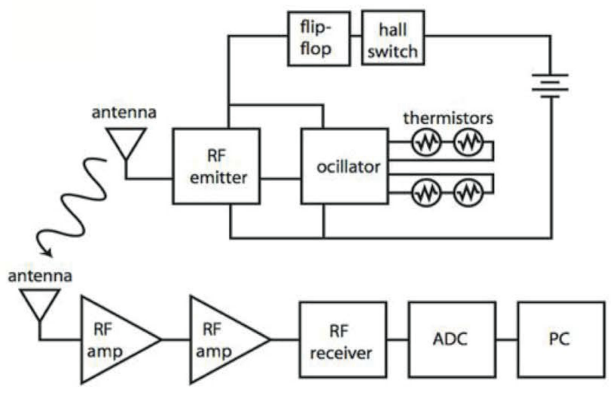

Figure 17. The smart particle measuring temperature uses four thermistors, placed directly in contact with the fluid, connected to a LMC555 timer to generate a square wave with frequency in the range $[22-26] \mathrm{kHz}$ depending on the flow temperature [Gasteuil et al., 2007; Shew et al., 2007]. A pair of RF emitter and receiver (MAX7044 and MAX1473 from Maxim Integrated Products) are used for frequency modulation and demodulation at $315 \mathrm{MHz}$. The emitter antenna is placed inside the capsule with emitting circuit tuned for emission at $315 \mathrm{MHz}$ using a variable capacitor. The signal is received by a fixed antenna, amplified and demodulated before acquisition at $10 \mathrm{MHz}$ with high speed DACQ. The slowly varying frequency of the square wave is then continuously measured using Labview standard library, then stored for further data analysis. Hall switch and flip-flop are used for turning on and off the particle approaching a magnet close to the particle in order to save battery when the experiment is not running.

Measurement in Rayleigh-Bénard convection. The particle was first used to investigate natural convection in a square tank with size $30 \mathrm{~cm}$ at high Rayleigh numbers
$R a \sim 10^{10}$. Figure 18(top) shows the time evolution of temperature along the particle trajectory with irregular oscillations caused by the motion of the particle crossing cold and hot regions in the vessel. Combining lagrangian temperature measurement and Particle Tracking Velocimetry, it becomes possible to study the correlations between position and temperature as shown in figure 18(bottom). As all kinematic quantities (velocity or acceleration) can be obtained from PTV data it is then possible to have informations about the turbulent heat flux $q=\left\langle v^{\prime} T^{\prime}\right\rangle$ (with $v^{\prime}$ and $T^{\prime}$ fluctuating velocity and temperature) in the whole experiment volume with insight form the role of plumes in heat transport [Gasteuil et al., 2007; Shew et al., 2007].
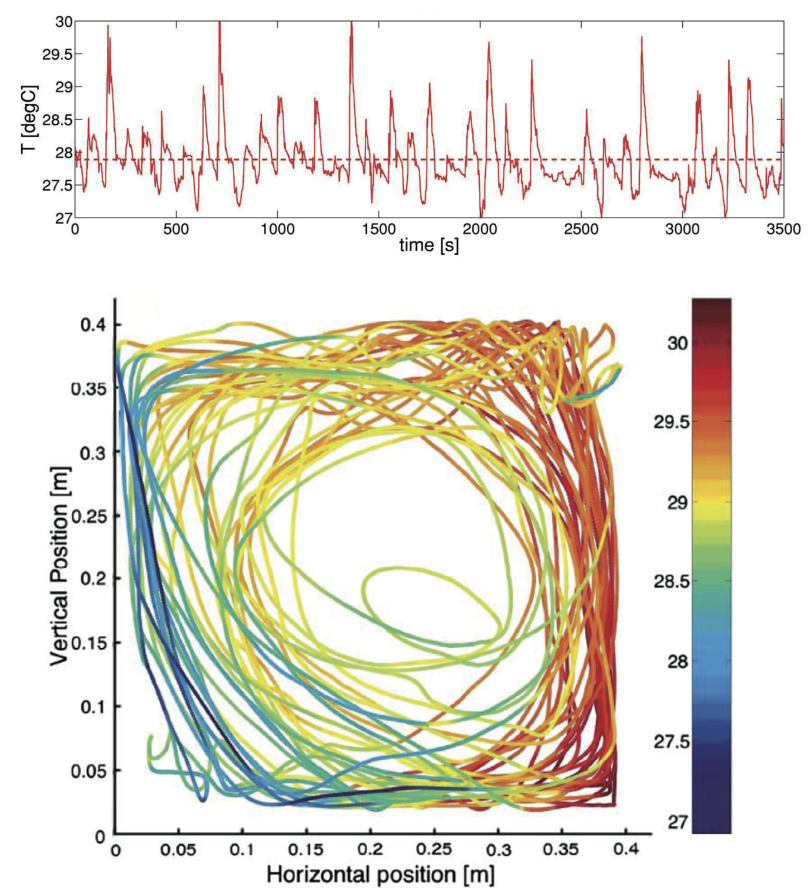

Figure 18. Top : Temporal evolution of temperature along a particle trajectory in turbulent Rayleigh Bénard convection with square aspect-ratio. Bottom : combined PTV-instrumented particle measurement showing the $2 \mathrm{~d}$ position of the particle $(X(t), Y(t))$ with local temperature $T(t)$ (see colorbar for for values of temperature in Celsius). Figure from [Shew et al., 2007].

\subsection{Further Lagrangian measurements.}

More recently the smart particle concept was extended to measurements of acceleration using numerical modulation and demodulation with suitable electronics [Zimmermann et al., 2012]. The apparatus was designed from the earlier work by Gasteuil et al. [Gasteuil, 2009], and built by smartINST S.A.S., an offspring company from CNRS and ENS de Lyon. It is a spherical particle with diameter $25 \mathrm{~mm}$ which embarks an autonomous circuit with 3D-acceleration sensor, a coin cell and a wireless transmission system. It transfers the measured data to a data acquisition center (smartCENTER) which decodes, processes, and stores the signal delivered by the smart particle. The smart particle and smartCENTER measure, display and store the three dimensional acceleration vectors acting on the particle as it is advected in the flow. The acceleration sensor is an 
ADXL 330 (Analog Device). The three axes of the ADXL 330 are decoupled and form an orthogonal coordinate system attached to the chip package. This arrangement yields a 3D measurement of acceleration, including gravity, with a full scale of $+/-3 \mathrm{~g}$. The sensor has to be calibrated to compute the physical accelerations from the voltages of the accelerometer.

Other sensors: the smart particle technology allows the transmition additional information originating from other sensors. Current developments aim at conductivity measurements which are of interest for salinity measurements and mixing issues in stratified flows.

\section{Conclusions and discussion}

We have reported here some of the latest developments in Lagrangian characterization of flows dynamics in laboratory experiments. Although the Lagrangian approach has been already identified by Taylor and Richardson as a relevant description of geophysical flows, it is only recently that technological progress have allowed to develop platforms with sufficient accuracy and resolution. Some of these techniques, including 3D-PTV and instrumented particles, have already been successfully used in model experiments with atmospheric and oceanographic motivations. But most of their advantages are still to be exploited. For instance the versatility of 3D-PTV, the ultimate temporal resolution of ELDV, the possibility to perform large scale atmospheric in situ measurements with acoustic tracking, the capacity to directly access Fourier modes of vorticity using acoustic scattering, the capacity of instrumented particles to measure not only kinematic quantities, but also heat transport, salinity, etc., show the many possible developments and advances that can still be expected from these techniques in addressing geophysical fluid dynamics issues.

Let us briefly summarize advantages and drawbacks of the different techniques. High resolution optical tracking has become one of the most accurate techniques in experimental fluid mechanics. It allows to track simultaneously hundreds of particles in 3D allowing to address central questions, related for instance to mixing and transport properties of flows. Its main drawback is the current cost high speed cameras. Acoustic tracking and ELDV, both based on Doppler velocimetry are more affordable techniques, though they are limited to the tracking of essentially one particle at a time and are therefore not adapted to multi-particle studies. Their main advantage lies in the fact that they give a direct measurement of particle velocity (and not particle position as in optical tracking) hence limiting the increase of noise induced by the differentiation of position to access velocity. Similarly, measuring acceleration of the particles requires only one differentiation step while second derivatives must be estimated from optical tracking. These techniques are therefore very accurate to investigate the Lagrangian dynamics of individual particles. Instrumented particles have ported further the capacity of investigation of Lagrangian properties of flows by giving access not only to kinematic properties (as velocity or acceleration) but also to a Lagrangian description of almost any physical quantity for which a relevant sensor can be embedded in the particle. The main drawback for the moment concerns the size of the particle which does not allow to probe scales smaller than about $1 \mathrm{~cm}$. Finally the acoustical vorticity measurement is unique of its kind as it gives a simple and accurate way to characterize the enstrophy spectrum of a flow, with intrinsic spectral resolution at a selected scale (including the smallest scales of the flow, which are hardly accessible with classical techniques as PIV).

Note that we have not reported here all the possible extensions and add-ons of these methods as for instance the use of digital holography [Salazar et al., 2008; Chareyron, 2009] which allows to track particles in 3D with one single camera, or the tracking of particles rotational dynamics [Zimmermann et al., 2011a, b; Klein et al., 2012] which allows to simultaneously investigate the translation and rotation of finite objects transported in a flow.

Experimental techniques in fluid mechanics are constantly improved, as new ideas combined with technological advances increase the resolution and the range of existing methods: cameras are for instance ever faster and sensors better resolved; miniaturization and reduction of power consumption of electronic components will allow to reduce the size of instrumented particles; an important breakthrough in high resolution optical tracking is expected in the coming years thanks to FPGA (Field Programmable Gate Array) technology which allows to process images on-board and hence to increase the effective data rate (for instance, particle detection could be done on-board and only the particle positions would be recorded). In this rapidly evolving context it is essential to promote an efficient interaction between fluid mechanics experimentalists and other communities, as geophysicists, in order to develop the appropriate instrumentation for Lab or field investigations.

\section{References}

Ayyalasomayajula, S., A. Gylfason, L. R. Collins, E. Bodenschatz, and Z. Warhaft, Lagrangian measurements of inertial particle accelerations in grid generated wind tunnel turbulence, Physical Review Letters, 97, 144,507, 2006.

Batchelor, G. K., Wave scattering due to turbulence, in Symposium on Naval-Hydrodynamics, edited by F. S. Sherman, pp. 403-429, National Academy of Sciences, Washington, 1957

Baudet, C., S. Ciliberto, and J.-F. Pinton, Spectral analysis of the von Karman flow using ultrasound scattering, Physical Review Letters, 67, 193-195, 1991 .

Bezaguet, A., et al., A cryogenic high Reynolds turbulence experiment at CERN, Adv. Cryo. Eng., 47, 136-144, 2002.

Bourgoin, M., N. T. Ouellette, H. T. Xu, J. Berg, and E. Bodenschatz, The role of pair dispersion in turbulent flow, Science, 311, $835-838,2006$

Bourgoin, M., N. M. Qureshi, C. Baudet, A. Cartellier, and C. Gagne, Turbulent transport of finite sized material particles, Journal of Physics: Conference Series, 318, 012,005, 2011.

Brown, R. D., Z. Warhaft, and G. A. Voth, Acceleration Statistics of Neutrally Buoyant Spherical Particles in Intense Turbulence, Physical Review Letters, 103, 2009.

Chareyron, D., Développement de méthodes instrumentales en vue de l'étude Lagrangienne de l'évaporation dans une turbulence homogène isotrope, Ph.D. thesis, Ecole Centrale de Lyon, 2009.

Chu, B. T., Non-Linear interactions in a viscous heat-conducting compressible gas, Journal of Fluid Mechanics, 3, $494-514,1958$. 
Colonius, T., S. K. Lele, and P. Moin, The scattering of sound waves by a vortex: numerical and analytical solutions, Journal of Fluid Mechanics, 260, 271-298, 1994.

Del Castello, L., and H. J. H. Clercx, Lagrangian acceleration of passive tracers in statistically steady rotating turbulence, Physical Review Letters, 10\%, 214,502, 2011.

Flandrin, P., Time-Frequency/Time-Scale Analysis, Academic Press, New York, 1998.

Gasteuil, Y., Instrumentation Lagrangienne en Turbulence: Mise en oeuvre et Analyse, Ph.D. thesis, ENS Lyon, 2009.

Gasteuil, Y., W. L. Shew, M. Gibert, F. Chillà, B. Castaing, and J.-F. Pinton, Lagrangian temperature, velocity, and local heat flux measurement in Rayleigh-B\{é\}nard convection, Phys. ${ }^{\sim}$ Rev. ${ }^{\sim}$ Lett., $99,234,302,2007$.

Klein, S., M. Gibert, and B. Antoine, Simultaneous 3D measurement of the translation and rotation of finite size particles and the flow field in a fully developed turbulent water flow ., ArXiv, p. arXiv:1205.2181v1, 2012.

Kodera, K., C. de Villedary, and R. Gendrin, A new method for the numerical analysis of nonstationary signals, Physics of the Earth and Planetary Interiors, 12, 142-150, 1976.

Kraichnan, R. H., The scattering of sound in a turbulent medium, J. Acoust. Soc. Am., 25, 1096-1104, 1953.

LaPorta, A., G. A. Voth, A. M. Crawford, J. Alexander, and E. Bodenschatz, Fluid particle accelerations in fuly developped turbulence, Nature, 409, 1017, 2001.

Llewellyn Smith, S. G., and R. Fort, Three dimensional acoustic scattering by vortical flows, Physics of Fluids, $13,2876-2889,2001$.

Lund, F., and C. Rojas, Ultrasound as a Probe of Turbulence, Physica D, 37, 508-514, 1989.

Mordant, N., Mesure lagrangienne en turbulence : mise en œuvre et analyse, Ph.D. thesis, Ecole Normale Supérieure de Lyon, 2001.

Mordant, N., P. Metz, O. Michel, and J.-F. Pinton, Measurement of Lagrangian Velocity in Fully Developed Turbulence, Physical Review Letters, 87, 214,501, 2001.

Mordant, N., J.-F. Pinton, and O. Michel, Time-resolved tracking of a sound scatterer in a complex flow: Nonstationary signal analysis and applications, Journal of the Acoustical Society of America, 112, 108-118, 2002.

Mordant, N., P. Metz, J. F. Pinton, and O. Michel, Acoustical technique for Lagrangian velocity measurement, Review of Scientific Instruments, 76, 25,105, 2005.

Ni, R., S.-D. Huang, and K.-Q. Xia, Lagrangian acceleration measurements in convective thermal turbulence, Journal of Fluid Mechanics, 692, 395-419, 2012.

Obukhov, A. M., Effect of weak inhomogeneities in the atmosphere on sound and light propagation, Izv. Akad. Nauk. Seriya Geofiz., 2, 155-165, 1953.

Ott, S., and J. Mann, An experimental investigation of the relative diffusion of particle pairs in three-dimensional turbulent flow, Journal of Fluid Mechanics, 422, 207-223, 2000.

Ouellette, N. T., H. Xu, and E. Bodenschatz, A quantitaive study of three-dimensional Lagrangian particle tracking algorithms, Experiments in Fluids, 39, 722-729, 2005.

Pietropinto, S., et al., Superconducting instrumentation for high Reynolds turbulence experiments with low temperature gaseous helium, Physica C, 386, 512-516, 1999.

Poulain, C., N. Mazellier, P. Gervais, Y. Gagne, and C. Baudet, Spectral vorticity and Lagrangian velocity measurements in turbulent jets, Flow, Turbulence and Combustion, 72, 245-271, 2004.

Qureshi, N., Experimental Investigation of Finite Sized Inertial Particles Dynamics in Wind Tunnel Grid Generated Turbulence, Ph.D. thesis, Université Joseph Fourier - Grenoble I, 2009.

Qureshi, N. M., M. Bourgoin, C. Baudet, A. Cartellier, and Y. Gagne, Turbulent transport of material particles: An experimental study of finite size effects, Physical Review Letters, 99, 2007.

Qureshi, N. M., U. Arrieta, C. Baudet, A. Cartellier, Y. Gagne, and M. Bourgoin, Acceleration statistics of inertial particles in turbulent flow, European Physical Journal B, 66, 531-536, 2008.

Richardson, L. F., Atmospheric Diffusion Shown on a Distance-Neighbour Graph, Proceedings of the Royal Society of London, Series A, 110, 709-737, 1926.

Salazar, J. P. L. C., J. de Jong, L. Cao, S. H. Woodward, H. Meng, and L. R. Collins, Experimental and numerical investigation of inertial particle clustering in isotropic turbulence, Journal of Fluid Mechanics, 600, 245-256, 2008.

Shew, W. L., Y. Gasteuil, M. Gibert, P. Metz, and J.-F. Pinton, Instrumented tracer for Lagrangian measurements in RayleighB $\{$ é\}nard convection, Rev. Sci. Instru., 78, 65,105, 2007.

Taylor, G. I., Diffusion by continuous movements, Proc. Lond. Math. Soc., 20, 196-212, 1922.

Tennekes, H., and J. L. Lumley, A first course in turbulence, MIT press, 1992.

Tsai, R., A versatile camera calibration technique for high accuracy $3 \mathrm{~d}$ machine vision metrology using off-the-shelf tv cameras and lenses, IEEE T. Robot. Autom., RA-3, 323, 1987.

Virant, M., and T. Dracos, $\{3 \mathrm{D}\}\{$ PTV $\}$ and its application on lagrangian motion, Measurement science and technology, 8, 1539-1552, 1997.

Volk, R., N. Mordant, G. Verhille, and J.-F. Pinton, Laser Doppler measurement of inertial particle and bubble accelerations in turbulence, European Physics Letters, 81, 34,002, 2008.

Volk, R., E. Calzavarini, E. Leveque, and J.-F. Pinton, Dynamics of inertial particles in a turbulent von Karman flow, Journal of Fluid Mechanics, 668, 223-235, 2011.

Voth, G. A., A. LaPorta, A. M. Crawford, J. Alexander, and E. Bodenschatz, Measurement of particle accelerations in fully developed turbulence, Journal of Fluid Mechanics, 469, 121-160, 2002.

$\mathrm{Xu}, \mathrm{H}$., and E. Bodenschatz, Motion of inertial particles with size larger than Kolmogorov scale in turbulent flows, Physica D, 237, 2095-2100, 2008.

Zimmermann, R., Y. Gasteuil, M. Bourgoin, R. Volk, A. Pumir, and J.-F. Pinton, Rotational Intermittency and Turbulence Induced Lift Experienced by Large Particles in a Turbulent Flow, Physical Review Letters, 106, 154,501, 2011a.

Zimmermann, R., Y. Gasteuil, M. Bourgoin, R. Volk, A. Pumir, and J.-F. Pinton, Tracking the dynamics of translation and absolute orientation of a sphere in a turbulent flow, Review of Scientific Instruments, 82, 33,906, $2011 \mathrm{~b}$.

Zimmermann, R., L. Fiabane, Y. Gasteuil, R. Volk, and J.-F. Pinton, Characterizing flows with an instrumented particle measuring Lagrangian accelerations, arXiv:1208.2809 [physics.flu-dyn], 2012.

M. Bourgoin, Laboratoire des Écoulements Géophysiques et Industriels, CNRS/UJF/GINP - Université de Grenoble, BP53 - 38041

Grenoble Cedex 9, France. (mickael.bourgoin@legi.grenoble-inp.fr)

J.-F. Pinton, CNRS UMR5672, Laboratoire de Physique de l'École normale supérieure de Lyon, 46 Allée d'Italie, 69007 Lyon, France.

R. Volk, CNRS UMR5672, Laboratoire de Physique de l'École nor male supérieure de Lyon, 46 Allée d'Italie, 69007 Lyon, France. 